Shock wave interaction with viscous wake in supersonic flow

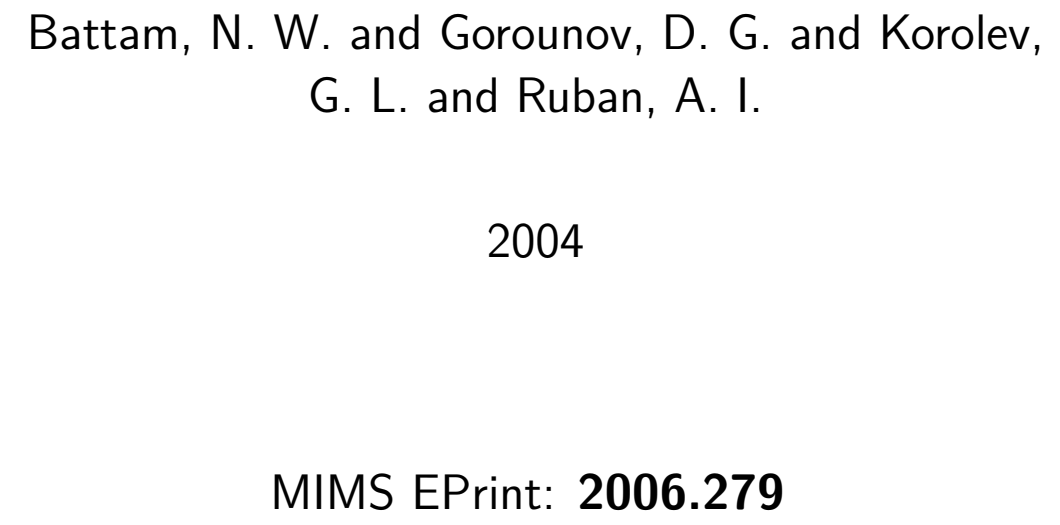

Battam, N. W. and Gorounov, D. G. and Korolev, G. L. and Ruban, A. I.

2004

MIMS EPrint: 2006.279

Manchester Institute for Mathematical Sciences

School of Mathematics

The University of Manchester

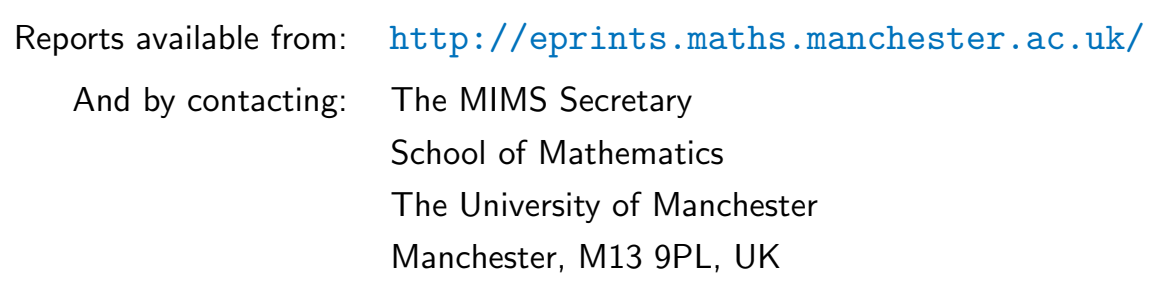

ISSN 1749-9097 


\title{
Shock wave interaction with a viscous wake in supersonic flow
}

\author{
By N. W. BATTAM, D. G. GOROUNOV, G. L. KOROLEV \\ AND A. I. RUBAN
}

Department of Mathematics, University of Manchester, Oxford Road, Manchester M13 9PL, UK

(Received 24 March 2003 and in revised form 3 December 2003)

A theoretical investigation of the breakdown of the viscous wake downstream of a flat plate in supersonic flow is performed in this paper based on the large Reynolds number $(R e \rightarrow \infty)$ asymptotic analysis of the Navier-Stokes equations. The breakdown is provoked by an oblique shock wave impinging on the wake a small distance $l_{s}$ downstream of the plate trailing edge. Two flow regimes are considered. In the first $l_{s}$ is assumed to be an $O\left(R^{-3 / 8}\right)$ quantity in which case the shock impinges on the wake within the region of viscous-inviscid interaction that is known to occupy a vicinity of the trailing edge with longitudinal extent of $O\left(R e^{-3 / 8}\right)$. Under these conditions the interaction process may be described by the equations of the triple-deck theory. To obtain a numerical solution of these equations we used a rapid matrix Thomas technique in conjunction with Newton iterations. The results of the calculations not only predict the wake breakdown near the shock location but also reveal a hysteresis behaviour of the flow as the shock is moved downstream, giving rise to three solution branches. The second part of the paper is concerned with the flow regime when the shock interacts with the wake further downstream of the trailing edge triple-deck region: $l_{s} \gg R e^{-3 / 8}$. In this case the fluid motion proves to be inviscid to leading order not only in the upper deck of the interaction region but also everywhere inside the wake. Due to this simplification the interaction problem can be reduced to a single integro-differential equation governing the pressure distribution along the interaction region. With known pressure the Bernoulli equation may be used to find the velocity field. The Bernoulli equation also allows us to formulate a simple criterion which may be used to predict the onset of wake breakdown. We found that viscosity becomes important again in a smaller vicinity of the breakdown point where the flow reversal takes place. It is remarkable that the viscous-inviscid interaction problem governing the flow in this vicinity admits an analytical solution.

\section{Introduction}

This study was undertaken as a first step in developing an asymptotic theory of vortex breakdown in supersonic flow. It was motivated by numerous experimental investigations of the phenomenon and an observation that the vortex breakdown proceeds very much in the same way as the boundary-layer separation provoked by an impinging shock wave.

The phenomenon of vortex breakdown has attracted significant attention during the last forty years. While a majority of publications in this field have been concerned with incompressible flows (see, for example, Benjamin 1962; Sarpkaya 1971; Hall 1972; Leibovich 1978, 1984; Delery 1994), a significant body of information has also been 
collected on the interaction of streamwise vortices with shock waves in supersonic flows. Gustintsev et al. (1969) and Zataloka et al. (1978) were, apparently, the first to notice that, when a streamwise vortex encounters a shock wave, a breakdown (also called 'vortex bursting') may be observed. If it happens, say, in front of an aircraft engine, then the quality of the intake flow can be seriously compromised. To model this situation Zataloka et al. (1978) investigated the interaction of a wing-tip vortex with a bow shock in front of a choked Pitot-type inlet.

Subsequently, detailed experiments on different aspects of supersonic vortex breakdown were conducted by Delery et al. (1984), Glotov (1989), Ivanyushkin et al. (1989), Metwally et al. (1989), Cattafester \& Settles (1992), Kalkhoran \& Sforza (1994), Kalkhoran (1994), Smart \& Kalkhoran (1995) and Kalkhoran et al. (1996, 1998). They observed that if a vortex is generated in a supersonic flow by, say, a tip of a wing and then after convecting some distance downstream intersects a bow shock wave, then a significant part of the flow upstream of the shock may be affected. In fact the vortex-shock interaction process proved to be dependent on the intensity of swirling motion in the vortex, the deficit of axial velocity at the vortex centre and the shock strength, i.e. the value by which the pressure jumps across the shock. If the shock is weak, then the vortex passes through the shock almost unaffected. However, on increasing the pressure jump the thickening of the vortex core becomes more pronounced, and the shock front starts to deform, curving towards the incoming vortex. The vortex breakdown takes place at a critical value of the pressure jump across the shock. Further strengthening of the shock results in the formation of a conical recirculation region. By this stage the bow shock is completely destroyed, being substituted by a conical shock with its apex situated at the point of breakdown. The apex moves upstream as the shock strength increases.

Starting with Gustintsev et al. (1969) and Zataloka et al. (1978) a number of authors commented that the supersonic vortex breakdown very much resembles the interaction of a shock wave with the boundary layer on a rigid body surface. This phenomenon was the focus of attention of many experimentalists (see, for example, a review by Chapman et al. 1956) starting with Ferri (1939). In a typical experiment the flow is composed of the boundary layer developing along a solid body, say a flat plate, which is aligned with the oncoming flow and a shock wave produced by a wedge installed above the plate. The shock impinges upon the boundary layer at some point $S$ on the plate surface, and the task is to study the perturbations produced in the flow due to the interaction of the shock and boundary layer.

The interest in this problem was mainly due to the fact that the observations showed an unexpected character of the flow. If one attempts to predict the flow behaviour based on the classical Prandtl (1904) theory of high Reynolds number flows then, as a first step of Prandtl's hierarchical procedure, the main body of the flow (where the fluid motion may be treated as inviscid) should be considered. At a supersonic speed the governing Euler equations are hyperbolic; they do not allow upstream propagation of perturbations. This suggests that the inviscid flow field should remain unperturbed everywhere in front of the incident shock. Turning next to the viscous boundary layer that forms along the body surface, it is known that Prandtl's equations for the boundary layer are parabolic, and therefore the flow in the boundary layer is also incapable of conducting any perturbation upstream of point $S$.

These theoretical arguments proved to fail completely in predicting the real behaviour of the flow. The experiments invariably showed that even when the shock was weak a thickening of the boundary layer started upstream of point $S$. The perturbations proved to be capable of propagating upstream through the boundary 
layer over a distance that exceeds significantly the thickness of the boundary layer. The boundary-layer separation was observed at a critical value of the pressure jump across the shock, and then the separation point started to move upstream as the shock strength was increased. It was further established that the boundary layer was perturbed even upstream of the separation point. Here an increase of pressure caused the streamlines in the boundary layer to deviate from the wall, giving rise to a secondary shock. Together with the primary shock it forms the so-called $\lambda$-structure.

There have been many attempts to give a theoretical explanation of the upstream influence through the boundary layer. A history of the development of ideas in this field was recently given by Lighthill (2000) who was the first to produce a consistent theory of the boundary-layer interaction with an oblique shock wave under an assumption that the shock was weak (see Lighthill 1953). Linear analysis of perturbations was used in this theory similar to that in the hydrodynamic stability theory. As a result it was shown, in particular, that the upstream influence of the shock extends over an $O\left(R e^{-3 / 8}\right)$ region; Re being the Reynolds number.

This development led ultimately to the creation of the asymptotic theory of viscousinviscid interaction also known as the triple-deck theory which gave a complete and systematic description of physical processes involved in the separation process. For the supersonic boundary-layer separation caused by an impinging shock or any other perturbation causing an abrupt pressure rise in the boundary layer (like a compression ramp or forward facing step) the theory was developed independently by Neiland (1969) and Stewartson \& Williams (1969). At the same time Messiter (1970) arrived independently at the triple-deck formulation in the course of an investigation of the incompressible flow near the trailing edge of a flat plate. Two years later Sychev (1972) demonstrated that the theory may be also used to describe a classical problem of the boundary-layer separation from a smooth body contour, say a circular cylinder, in incompressible flow. All these works were based on asymptotic analysis of the Navier-Stokes equations with $R e \rightarrow \infty$ using the principle of matched asymptotic expansions.

Later it became clear that the triple-deck viscous-inviscid interaction theory governs a wide variety of fluid dynamic phenomena, including boundary-layer separation in subsonic, transonic and supersonic flows, development of different modes of instabilities, bifurcation and possible hysteresis in high Reynolds number flows. Application of the theory to incompressible separated flows is presented in a monograph by Sychev et al. (1998) which describes the separation from a smooth body surface, incipient and large-scale separations at angular points of the body contour, separation at the trailing edge of a thin aerofoil appearing as a result of increase of the angle of attack or the aerofoil thickness, leading-edge separation, etc.

In the first part of this paper $(\S \S 2-4)$ we shall use the triple-deck theory to describe the process of the interaction of an oblique shock wave with the wake downstream of a flat plate. The symmetrical problem of supersonic flow past a flat plate free of shock waves was considered by Daniels (1974). He found that the fluid in the boundary layer is subject to an acceleration when moving from the plate surface into the wake. No flow separation could be expected in this flow, which allowed a relatively simple numerical treatment of the flow in the triple-deck region occupying an $O\left(R e^{-3 / 8}\right)$ vicinity of the trailing edge. We add to the Daniels' (1974) formulation a shock wave impinging on the wake at a point $S$. The distance $l_{s}$ between point $S$ and the trailing edge of the flat plate is assumed to be an $O\left(R e^{-3 / 8}\right)$ quantity allowing the triple-deck theory to remain applicable. However, from a numerical point of view the problem becomes significantly more complicated. We shall see that the impinging 


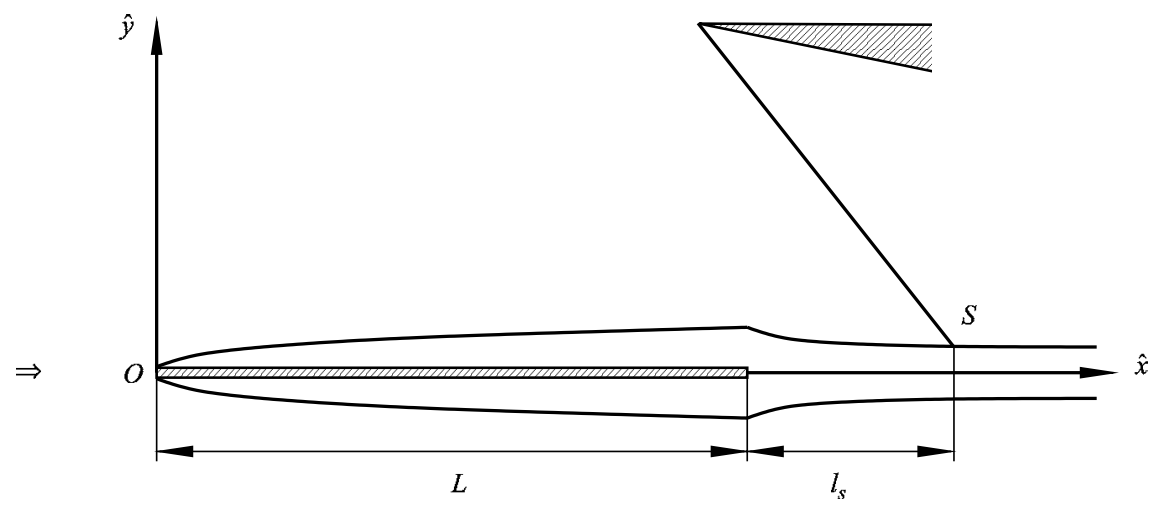

FIGURE 1. The flow layout.

shock not only can cause the breakdown of the wake near point $S$ but also the solution bifurcates, with one of the branches representing the flow with boundarylayer separation on the plate surface. An advanced numerical method based on a rapid matrix Thomas technique has been used to study the flow behaviour. This method is described in $\S 2$, with the results of calculations presented in $\S 3$.

The second part of the paper $(\S 5$ and $\S 6)$ is concerned with the flow regime when the shock wave is moved further downstream, i.e. $l_{s} \gg R e^{-3 / 8}$. An incompressible version of the problem was earlier considered by Sychev (1978); see also chapter 5 in Sychev et al. (1998). Our analysis in $\S 5$ shows that the deceleration of the flow in the wake, caused by the impinging shock, is predominantly inviscid. Due to this simplification the interaction problem can be reduced to a single integro-differential equation governing the pressure rise prior to the wake breakdown. The viscosity becomes important again in a smaller vicinity of the point of flow reversal $(\$ 6)$.

\section{Formulation of the problem}

Let a flat plate be placed in supersonic flow of a perfect gas such that the plate surface is parallel to the free-stream velocity vector. Let further a shock wave be generated be a wedge installed above the plate as shown in figure 1, and impinges upon the wake at point $S$.

We denote the plate length by $L$; the velocity, density, viscosity and pressure in the unperturbed flow upstream of the plate are denoted by $U_{\infty}, \rho_{\infty}, \mu_{\infty}$ and $p_{\infty}$ respectively. To study the flow we shall use Cartesian coordinate system $\hat{x} O \hat{y}$ with $\hat{x}$ measured parallel to the plate from its leading edge, and $\hat{y}$ in the perpendicular direction. The velocity components in these coordinates are denoted by $\hat{u}$ and $\hat{v}$. We also need to introduce the gas density $\hat{\rho}$, pressure $\hat{p}$, enthalpy $\hat{h}$ and viscosity $\hat{\mu}$. The 'hat' is used here for dimensional variables. The non-dimensional variables are introduced as

$$
\begin{gathered}
\hat{u}=U_{\infty} u, \quad \hat{v}=U_{\infty} v, \quad \hat{\rho}=\rho_{\infty} \rho, \\
\hat{p}=p_{\infty}+\rho_{\infty} U_{\infty}^{2} p, \quad \hat{h}=U_{\infty}^{2} h, \quad \hat{\mu}=\mu_{\infty} \mu, \\
\hat{x}=L x, \quad \hat{y}=L y .
\end{gathered}
$$


In what follows an asymptotic analysis of the Navier-Stokes equations will be conducted assuming that the Reynolds number

$$
R e=\frac{\rho_{\infty} U_{\infty} L}{\mu_{\infty}} \rightarrow \infty .
$$

\subsection{Boundary-layer flow}

Both in the boundary layer on the plate surface and in the wake downstream of the plate trailing edge the solution of the Navier-Stokes equations may be represented in the form of the asymptotic expansions

$$
\left.\begin{array}{lll}
u(x, y ; R e) & =U_{0}(x, Y)+\cdots, & v(x, y ; R e)=R e^{-1 / 2} V_{0}(x, Y)+\cdots, \\
\rho(x, y ; R e)=\rho_{0}(x, Y)+\cdots, & p(x, y ; R e)=R e^{-1 / 2} P_{1}(x, Y)+\cdots, \\
h(x, y ; R e)=h_{0}(x, Y)+\cdots, & \mu(x, y ; R e)=\mu_{0}(x, Y)+\cdots,
\end{array}\right\}
$$

where the coordinate normal to the wall is scaled as

$$
y=R e^{-1 / 2} Y .
$$

Substitution of (2.1) into the Navier-Stokes equations leads to the classical boundarylayer equations

$$
\begin{aligned}
\rho_{0} U_{0} \frac{\partial U_{0}}{\partial x}+\rho_{0} V_{0} \frac{\partial U_{0}}{\partial Y} & =\frac{\partial}{\partial Y}\left(\mu_{0} \frac{\partial U_{0}}{\partial Y}\right), \\
\rho_{0} U_{0} \frac{\partial h_{0}}{\partial x}+\rho_{0} V_{0} \frac{\partial h_{0}}{\partial Y} & =\frac{1}{\operatorname{Pr}} \frac{\partial}{\partial Y}\left(\mu_{0} \frac{\partial h_{0}}{\partial Y}\right)+\mu_{0}\left(\frac{\partial U_{0}}{\partial Y}\right)^{2}, \\
\frac{\partial\left(\rho_{0} U_{0}\right)}{\partial x}+\frac{\partial\left(\rho_{0} V_{0}\right)}{\partial Y} & =0, \\
h_{0} & =\frac{1}{(\gamma-1) M_{\infty}^{2}} \frac{1}{\rho_{0}} .
\end{aligned}
$$

Here $\operatorname{Pr}$ is the Prandtl number, $\gamma$ is the specific heat ratio and $M_{\infty}$ is the free-stream Mach number defined as

$$
M_{\infty}=\frac{U_{\infty}}{a_{\infty}}, \quad a_{\infty}=\sqrt{\gamma \frac{p_{\infty}}{\rho_{\infty}}} .
$$

We shall assume that $M_{\infty}$ is a finite quantity greater than one.

Equations (2.2) should be solved with the free-stream conditions at the leading edge of the flat plate

$$
U_{0}=1, \quad h_{0}=\frac{1}{(\gamma-1) M_{\infty}^{2}} \quad \text { at } \quad x=0, \quad Y \in[0, \infty)
$$

as well as at the outer edge of the boundary layer including the wake

$$
U_{0}=1, \quad h_{0}=\frac{1}{(\gamma-1) M_{\infty}^{2}} \quad \text { at } \quad Y=\infty, \quad x \in[0, \infty) .
$$

On the plate surface the no-slip condition holds:

$$
U_{0}=V_{0}=0 \quad \text { at } \quad Y=0, \quad x \in[0,1] .
$$


It should be supplemented with a thermal condition. For example, we can assume that the wall temperature is given as a function of $x$, i.e.

$$
h_{0}=F(x) \quad \text { at } \quad Y=0, \quad x \in[0,1]
$$

or the wall is thermally isolated

$$
\frac{\partial h_{0}}{\partial Y}=0 \quad \text { at } \quad Y=0, \quad x \in[0,1] .
$$

Boundary-value problem (2.2)-(2.6) admits a self-similar solution for thermally isolated wall $(2.6 b)$ as well as in the case when the wall temperature is known to be constant, i.e. function $F(x)$ in $(2.6 a)$ does not depend on $x$. However, we do not need to restrict ourselves to these particular flow conditions. If they are not satisfied then the boundary-value problem (2.2)-(2.6) may be solved numerically. To proceed further we only need to know that the solution remains smooth when the trailing edge of the plate is approached. Therefore we shall assume that the sought functions $U_{0}, h_{0}, \rho_{0}$ and $\mu_{0}$ may be represented in the form of Taylor expansions

$$
\left.\begin{array}{l}
U_{0}(x, Y)=U_{00}(Y)+(-s) U_{01}(Y)+\cdots \\
h_{0}(x, Y)=h_{00}(Y)+(-s) h_{01}(Y)+\cdots \\
\rho_{0}(x, Y)=\rho_{00}(Y)+(-s) \rho_{01}(Y)+\cdots \\
\mu_{0}(x, Y)=\mu_{00}(Y)+(-s) \mu_{01}(Y)+\cdots
\end{array}\right\} \quad \text { as } \quad s=x-1 \rightarrow 0^{-} .
$$

The leading-order terms in (2.7) exhibit the following behaviour near the plate surface

$$
\left.\begin{array}{l}
U_{00}(Y)=\lambda Y+\cdots \\
h_{00}(Y)=h_{w}+\cdots \\
\rho_{00}(Y)=\rho_{w}+\cdots \\
\mu_{00}(Y)=\mu_{w}+\cdots
\end{array}\right\} \quad \text { as } \quad Y \rightarrow 0
$$

where $\lambda, h_{w}, \rho_{w}, \mu_{w}$ are positive constants representing the dimensionless skin friction, enthalpy, density and viscosity on the wall surface.

\subsection{Triple-deck region}

A vicinity of the trailing edge of the plate is known to be occupied by the viscousinviscid interaction region where the boundary layer and external inviscid flow are mutually dependent on one another. For supersonic flow past a flat plate it was first studied by Daniels (1974). Based on the asymptotic analysis of the NavierStokes equations with $R e \rightarrow \infty$ he demonstrated that similar to the corresponding incompressible flow (Messiter 1970) the region of interaction is $O\left(R e^{-3 / 8}\right)$ long and has a three-tiered structure shown in figure 2. It is composed of the viscous sublayer (region 1 in figure 2), the main part of the boundary layer (region 2) and an inviscid potential flow region 3 situated outside the boundary layer.

The characteristic thickness of the viscous sublayer is estimated as being an $O\left(R e^{-5 / 8}\right)$ quantity, so that it occupies an $O\left(R e^{-1 / 8}\right)$ portion of the boundary layer and is comprised of the stream filaments immediately adjacent to the wall. The flow velocity in this region is $O\left(R e^{-1 / 8}\right)$ relative to the free-stream velocity, and due to the slow motion of gas here the flow exhibits high sensitivity to pressure variations. Even a small variation of pressure along the wall may cause significant deceleration/acceleration of fluid particles there. As a result the flow filaments change their thickness leading to a deformation of streamlines. This process is termed the displacement effect of the boundary layer. Quantitatively it may be characterized by the angle $\vartheta=\arctan (v / u)$ made by the velocity vector with the $x$-axis. 


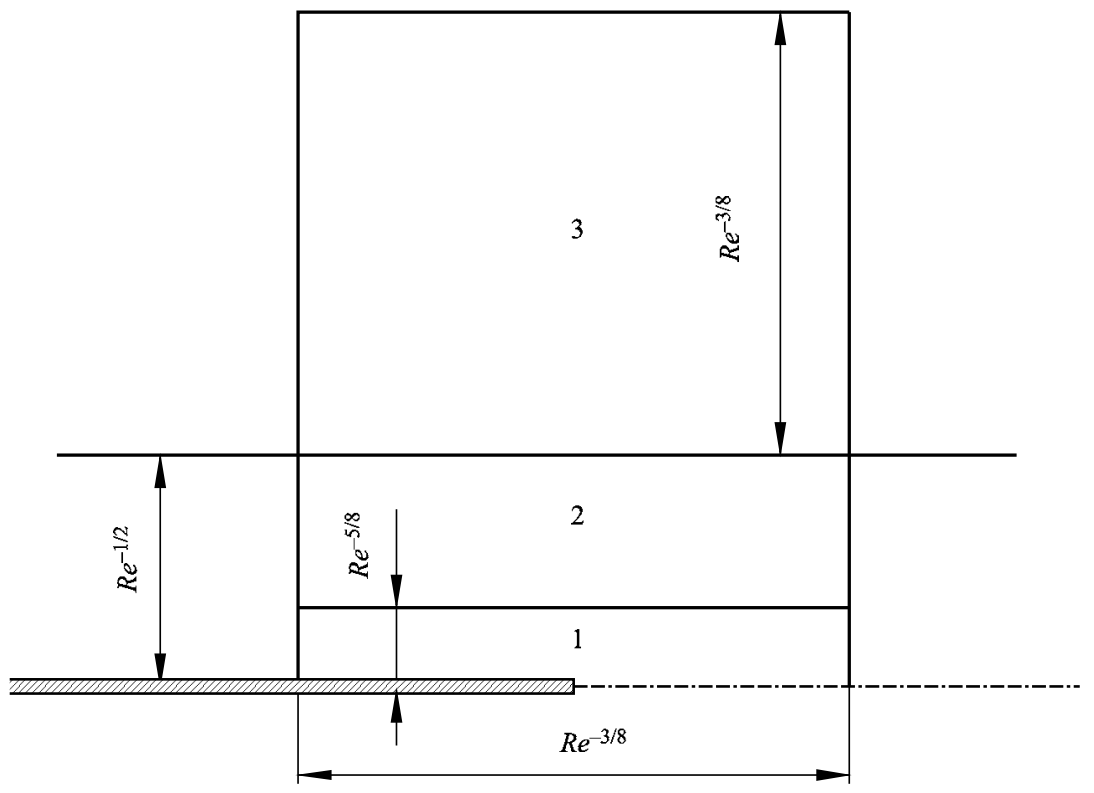

FIGURE 2. Three-tiered structure of the interaction region.

The main part of the boundary layer, the middle tier of the interactive structure, represents a continuation of the conventional boundary layer developing along the plate. Its thickness is estimated as $O\left(R e^{-1 / 2}\right)$ and the velocity is an order-one quantity. The flow in this tier is significantly less sensitive to the pressure variations. It does not produce any noticeable contribution to the displacement effect of the boundary layer, which means that all the streamlines in the middle tier are parallel to each other and carry the deformation produced by the displacement effect of the viscous sublayer.

Finally, the upper tier is situated in the potential flow region outside the boundary layer. It serves to 'convert' the perturbations in the form of the streamlines into perturbations of pressure. These are then transmitted through the main part of the boundary layer back to the sublayer. We shall assume here that the shock impinges upon the boundary layer within the interaction region, i.e. $l_{s}=O\left(R e^{-3 / 8}\right)$ (see figure 1$)$. As the velocity in the viscous sublayer $u=O\left(R e^{-1 / 8}\right)$ the breakdown of the wake may be expected to take place when the pressure rise in the impinging shock reaches a critical value of order $\Delta p \sim u^{2} \sim R e^{-1 / 4}$.

It should be noted that the trailing edge flow with no impinging shock waves considered by Daniels (1974) was symmetrical with respect to the $x$-axis. This allowed him to restrict the analysis to the upper half of the interaction structure shown figure 2. With the shock wave present one has to keep in mind that in reality the viscous sublayer (region 1) extends over a distance $y \sim R e^{-5 / 8}$ into the flow below the plate. The boundary layer on the lower side of the plate forms region $2^{\prime}$ which is similar to region 2, and further below lies potential supersonic flow region $3^{\prime}$.

Asymptotic analysis of the Navier-Stokes equations in region 1 is based on the limit

$$
X_{*}=\frac{x-1}{R e^{-3 / 8}}=O(1), \quad Y_{*}=\frac{y}{R e^{-5 / 8}}=O(1), \quad R e \rightarrow \infty .
$$

Since the flow in this region is slow $\left(u \sim R e^{-1 / 8}\right)$, it can be treated as incompressible to the leading order. Based on the matching with the boundary-layer solution (2.7), 
(2.8) one can conclude that the gas density equals $\rho_{w}$ and viscosity $\mu_{w}$ throughout region 1 . The velocity components $u, v$ and pressure $p$ are represented in the form of asymptotic expansions

$$
\left.\begin{array}{l}
u(x, y ; R e)=R e^{-1 / 8} U^{*}\left(X_{*}, Y_{*}\right)+\cdots, \\
v(x, y ; R e)=R e^{-3 / 8} V^{*}\left(X_{*}, Y_{*}\right)+\cdots, \\
p(x, y ; R e)=R e^{-1 / 4} P^{*}\left(X_{*}, Y_{*}\right)+\cdots
\end{array}\right\}
$$

Substitution of (2.9) into the Navier-Stokes equations leads to the Prandtl boundarylayer equations

$$
\begin{aligned}
\rho_{w} U^{*} \frac{\partial U^{*}}{\partial X_{*}}+\rho_{w} V^{*} \frac{\partial U^{*}}{\partial Y_{*}} & =-\frac{\mathrm{d} P^{*}}{\mathrm{~d} X_{*}}+\mu_{w} \frac{\partial^{2} U^{*}}{\partial Y_{*}^{2}}, \\
\frac{\partial U^{*}}{\partial X_{*}}+\frac{\partial V^{*}}{\partial Y_{*}} & =0 .
\end{aligned}
$$

These should be solved with the no-slip condition on both sides of the plate

$$
U^{*}=V^{*}=0 \quad \text { at } \quad Y_{*}= \pm 0, \quad X_{*} \leqslant 0
$$

and the matching condition with the solution (2.1), (2.7), (2.8) in boundary-layer upstream of the interaction region

$$
U^{*}=\left\{\begin{array}{lll}
\lambda Y_{*}+\cdots & \text { as } \quad X_{*} \rightarrow-\infty, \quad Y_{*} \geqslant 0, \\
-\lambda Y_{*}+\cdots & \text { as } \quad X_{*} \rightarrow-\infty, \quad Y_{*} \leqslant 0 .
\end{array}\right.
$$

Here the symmetry of the boundary-layer flow on the upper and lower sides of the plate has been taken into account.

It may be shown (see the Appendix) that a solution of the boundary-layer equations (2.10) subject to the initial conditions (2.12) exhibits the following behaviour at the upper and lower edges of the viscous sublayer (region 1)

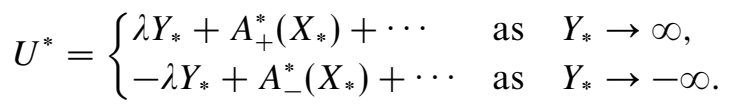

Functions $A_{+}^{*}\left(X_{*}\right)$ and $A_{-}^{*}\left(X_{*}\right)$ are termed the displacement functions. The reason for this may be explained by using (2.13) in the continuity equation $(2.10 b)$. We find

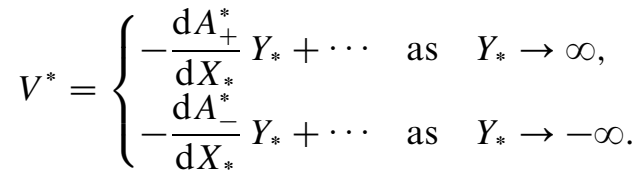

Combining (2.9), (2.13) and (2.14) together shows that the streamline slope angle at the upper and lower edges of the viscous sublayer

$$
\begin{aligned}
& \vartheta=\arctan \left(\frac{v}{u}\right)=R e^{-1 / 4} \frac{V^{*}}{U^{*}}+\cdots \\
& =\left\{\begin{array}{l}
R e^{-1 / 4}\left(-\frac{1}{\lambda} \frac{\mathrm{d} A_{+}^{*}}{\mathrm{~d} X_{*}}\right)+\cdots \quad \text { as } \quad Y_{*} \rightarrow \infty, \\
R e^{-1 / 4}\left(\frac{1}{\lambda} \frac{\mathrm{d} A_{-}^{*}}{\mathrm{~d} X_{*}}\right)+\cdots \quad \text { as } \quad Y_{*} \rightarrow-\infty .
\end{array}\right.
\end{aligned}
$$

Of course, functions $A_{+}^{*}\left(X_{*}\right)$ and $A_{-}^{*}\left(X_{*}\right)$ are not known in advance. Instead they have to be calculated by solving the problem of viscous-inviscid interaction. To 
complete the formulation of this problem we need to supplement (2.10)-(2.13) with the interaction law that serves to relate the induced pressure $P^{*}$ to $A_{+}^{*}\left(X_{*}\right)$ and $A_{-}^{*}\left(X_{*}\right)$. We note that the streamline slope $\vartheta$ does not change across the main part of the boundary layer, shown as region 2 in figure 2, and its counterpart, region $2^{\prime}$, situated in the flow below the plate. This means that formulae (2.15) prove to be valid at the 'bottom' of region 3 and at the 'top' of region $3^{\prime}$ where they can be used to formulate the boundary conditions for the equations governing the flow outside the boundary layer.

Guided by (2.15) we seek the solution in regions 3 and $3^{\prime}$ in the form of asymptotic expansions

$$
\left.\begin{array}{l}
u(x, y ; R e)=1+R e^{-1 / 4} u_{1}\left(X_{*}, y_{*}\right)+\cdots, \\
v(x, y ; R e)=R e^{-1 / 4} u_{1}\left(X_{*}, y_{*}\right)+\cdots, \\
\rho(x, y ; R e)=1+R e^{-1 / 4} \rho_{1}\left(X_{*}, y_{*}\right)+\cdots, \\
p(x, y ; R e)=R e^{-1 / 4} p_{1}\left(X_{*}, y_{*}\right)+\cdots, \\
h(x, y ; R e)=\frac{1}{(\gamma-1) M_{\infty}^{2}}+R e^{-1 / 4} h_{1}\left(X_{*}, y_{*}\right)+\cdots
\end{array}\right\}
$$

Here $X_{*}, y_{*}$ are scaled coordinates

$$
x=1+R e^{-1 / 4} X_{*}, \quad y=R e^{-1 / 4} y_{*},
$$

assumed to be order one quantities in regions 3 and $3^{\prime}$.

Substitution of (2.16) into the Navier-Stokes equations yields

$$
\begin{aligned}
\frac{\partial u_{1}}{\partial X_{*}} & =-\frac{\partial p_{1}}{\partial X_{*}}, \\
\frac{\partial v_{1}}{\partial X_{*}} & =-\frac{\partial p_{1}}{\partial y_{*}}, \\
\frac{\partial h_{1}}{\partial X_{*}} & =\frac{\partial p_{1}}{\partial X_{*}}, \\
\frac{\partial u_{1}}{\partial X_{*}}+\frac{\partial \rho_{1}}{\partial X_{*}}+\frac{\partial v_{1}}{\partial y_{*}} & =0, \\
h_{1} & =\frac{\gamma}{\gamma-1} p_{1}-\frac{1}{(\gamma-1) M_{\infty}^{2}} \rho_{1} .
\end{aligned}
$$

Eliminating $u_{1}, v_{1}, \rho_{1}$ and $h_{1}$ from (2.17) one can easily see that the pressure perturbations in regions 3 and $3^{\prime}$ are governed by the wave equation

$$
\left(M_{\infty}^{2}-1\right) \frac{\partial^{2} p_{1}}{\partial X_{*}^{2}}-\frac{\partial^{2} p_{1}}{\partial y_{*}^{2}}=0 .
$$

The boundary condition for this equation is formulated by setting $y_{*}=0$ in $(2.17 b)$,

$$
\left.\frac{\partial p_{1}}{\partial y_{*}}\right|_{y_{*}=0}=-\left.\frac{\partial v_{1}}{\partial X_{*}}\right|_{y_{*}=0} .
$$

The distribution of $v_{1}\left(X_{*}, 0\right)$ at the 'bottom' of region 3 and at the 'top' of region $3^{\prime}$ may be found through matching of the streamline slop angle $\vartheta$. In regions 2 and $2^{\prime}$ (see figure 2) it is given by (2.15). Using (2.16), we can see that in regions 3 and $3^{\prime}$

$$
\vartheta=\frac{v}{u}=R e^{-1 / 4} v_{1}\left(X_{*}, y_{*}\right)+\cdots,
$$


and therefore, the matching condition states

$$
\left.v_{1}\right|_{y_{*}=0+}=-\frac{1}{\lambda} \frac{\mathrm{d} A_{+}^{*}}{\mathrm{~d} X_{*}},\left.\quad v_{1}\right|_{y_{*}=0-}=\frac{1}{\lambda} \frac{\mathrm{d} A_{-}^{*}}{\mathrm{~d} X_{*}} .
$$

The general solution of (2.18) has the form

$$
p_{1}=f(\xi)+g(\eta)
$$

where $f$ and $g$ are arbitrary function of

$$
\xi=X_{*}-\sqrt{M_{\infty}^{2}-1} y_{*} \text { and } \eta=X_{*}+\sqrt{M_{\infty}^{2}-1} y_{*},
$$

respectively. The flow in region $3^{\prime}$ is assumed to be free of any perturbations except those produced by the displacement effect of the boundary layer. Therefore, function $f(\xi)$ which describes the pressure waves impinging upon the boundary layer, should be excluded from (2.21). Function $g(\eta)$ is easily found by substituting (2.21) and the second of equations (2.20) into (2.19). This yields

$$
g\left(x_{*}\right)=-\frac{1}{\lambda \sqrt{M_{\infty}^{2}-1}} \frac{\mathrm{d} A_{-}^{*}}{\mathrm{~d} X_{*}} .
$$

It remains to set $y_{*}=0$ in $(2.21)$ and we will find that the pressure induced by the supersonic flow in region $3^{\prime}$ at the lower edge of the boundary layer (including the wake) is calculated as

$$
P_{-}^{*}=-\frac{1}{\lambda \sqrt{M_{\infty}^{2}-1}} \frac{\mathrm{d} A_{-}^{*}}{\mathrm{~d} X_{*}} .
$$

In region 3, which is situated above the boundary layer (see figure 2), the role of functions $f(\xi)$ and $g(\eta)$ in (2.21) is different. Now $g(\eta)$ represents perturbations produced by an external source situated above the plate and upstream of the interaction region. In particular, a wedge may be used to generate a shock wave which then impinges upon the boundary layer. In this case function $g(\eta)$ should be written as

$$
g(\eta)=\bar{P}_{s} H\left(\eta-\bar{X}_{s}\right)
$$

Here $H$ is the Heaviside function,

$$
H(\eta)= \begin{cases}0 & \text { if } \quad \eta<0 \\ 1 & \text { if } \quad \eta \geqslant 0\end{cases}
$$

We assume that the pressure jump across the shock $\Delta p=R e^{-1 / 4} \bar{P}_{s}$ with $\bar{P}_{s}$ being an order-one parameter. We further assume that the shock impinges upon the wake (see figure 1) at a position $l_{s}=R e^{-3 / 8} \bar{X}_{s}$. This gives rise to the second parameter $\bar{X}_{s}$ in the above formula for $g(\eta)$.

In order to find function $f(\xi)$ we substitute (2.21) together with the first of equations (2.20) into (2.19). We find

$$
f\left(X_{*}\right)=g\left(X_{*}\right)-\frac{1}{\lambda \sqrt{M_{\infty}^{2}-1}} \frac{\mathrm{d} A_{+}^{*}}{\mathrm{~d} X_{*}} .
$$

Setting $y_{*}=0$ in (2.21) and using (2.23) and (2.24), we arrive at a conclusion that the pressure at the upper edge of the boundary layer

$$
P_{+}^{*}=2 \bar{P}_{s} H\left(X_{*}-\bar{X}_{s}\right)-\frac{1}{\lambda \sqrt{M_{\infty}^{2}-1}} \frac{\mathrm{d} A_{+}^{*}}{\mathrm{~d} X_{*}^{*}} .
$$


To close the interaction problem it remains to note that the pressure does not change across the boundary layer, and therefore

$$
P_{+}^{*}=P_{-}^{*} \text { for all } X_{*} \geqslant 0 .
$$

We shall now demonstrate that using new curvilinear coordinates

$$
\bar{X}=X_{*}, \quad \bar{Y}=Y_{*}-F(\bar{X})
$$

the interaction problem (2.10)-(2.13), (2.22)-(2.26) may be recast into a form that represents a symmetrical flow near the trailing edge with two shock waves, of equal strength, impinging from above and below upon the wake at point $\bar{X}_{s}$. According to the Prandtl transposition theorem, the boundary-layer equations (2.10) are invariant with respect to the coordinate transformation (2.27) with arbitrary function $F(\bar{X})$ provided that the sought fluid dynamic functions are transformed as

$$
\left.\begin{array}{l}
U^{*}=\bar{U}, \quad V^{*}=\bar{V}+\frac{\mathrm{d} F}{\mathrm{~d} \bar{X}} \bar{U}, \quad P^{*}=\bar{P}, \\
A_{+}^{*}=\bar{A}_{+}-\lambda F, \quad A_{-}^{*}=\bar{A}_{-}+\lambda F .
\end{array}\right\}
$$

Indeed, substitution of (2.27), (2.28) into (2.10) results in

$$
\begin{gathered}
\rho_{w} \bar{U} \frac{\partial \bar{U}}{\partial \bar{X}}+\rho_{w} \bar{V} \frac{\partial \bar{U}}{\partial \bar{Y}}=-\frac{\mathrm{d} \bar{P}}{\mathrm{~d} \bar{X}}+\mu_{w} \frac{\partial^{2} \bar{U}}{\partial \bar{Y}^{2}}, \\
\frac{\partial \bar{U}}{\partial \bar{X}}+\frac{\partial \bar{V}}{\partial \bar{Y}}=0 .
\end{gathered}
$$

If we assume that $F(\bar{X})=0$ for all $\bar{X} \leqslant 0$ then the no-slip conditions (2.11) remain unchanged

$$
\bar{U}=\bar{V}=0 \quad \text { at } \quad \bar{Y}= \pm 0, \quad \bar{X} \leqslant 0 .
$$

The initial conditions (2.11) preserve their form

$$
\bar{U}=\left\{\begin{array}{lll}
\lambda \bar{Y}_{*}+\cdots & \text { as } \quad \bar{X} \rightarrow-\infty, \quad \bar{Y} \geqslant 0, \\
-\lambda \bar{Y}_{*}+\cdots & \text { as } \quad \bar{X} \rightarrow-\infty, \quad \bar{Y} \leqslant 0,
\end{array}\right.
$$

provided that $F(\bar{X}) \rightarrow 0$ as $\bar{X} \rightarrow-\infty$.

The conditions at the lower and upper edges of the viscous sublayer (2.13) are invariant with respect to transformations (2.27), (2.28) for any choice of function $F(\bar{X})$ :

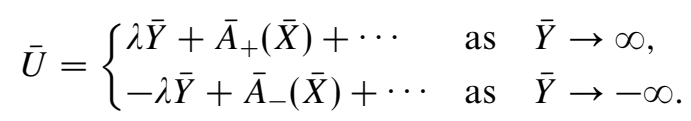

The interaction law (2.22), (2.25) is now written in the form

$$
\begin{aligned}
& \bar{P}_{-}=-\frac{1}{\lambda \sqrt{M_{\infty}^{2}-1}}\left(\frac{\mathrm{d} \bar{A}_{-}}{\mathrm{d} \bar{X}}+\lambda \frac{\mathrm{d} F}{\mathrm{~d} \bar{X}}\right), \\
& \bar{P}_{+}=2 \bar{P}_{s} H\left(\bar{X}-\bar{X}_{s}\right)-\frac{1}{\lambda \sqrt{M_{\infty}^{2}-1}}\left(\frac{\mathrm{d} \bar{A}_{+}}{\mathrm{d} \bar{X}}-\lambda \frac{d F}{d \bar{X}}\right) .
\end{aligned}
$$

Therefore, if we choose

$$
F(\bar{X})= \begin{cases}0 & \text { if } \bar{X}<\bar{X}_{s}, \\ -\sqrt{M_{\infty}^{2}-1} \bar{P}_{s}\left(\bar{X}-\bar{X}_{s}\right) & \text { if } \bar{X} \geqslant \bar{X}_{s},\end{cases}
$$


then the interaction law takes the form

$$
\begin{aligned}
& \bar{P}_{-}=\bar{P}_{s} H\left(\bar{X}-\bar{X}_{s}\right)-\frac{1}{\lambda \sqrt{M_{\infty}^{2}-1}} \frac{\mathrm{d} \bar{A}_{-}}{\mathrm{d} \bar{X}}, \\
& \bar{P}_{+}=\bar{P}_{s} H\left(\bar{X}-\bar{X}_{s}\right)-\frac{1}{\lambda \sqrt{M_{\infty}^{2}-1}} \frac{\mathrm{d} \bar{A}_{+}}{\mathrm{d} \bar{X}},
\end{aligned}
$$

and all the equations (2.29)-(2.33) involved in the formulation of the interaction problem prove to be symmetrical with respect to the $\bar{X}$-axis.

The physical meaning of this result is quite simple. We have demonstrated that the new longitudinal coordinate $\bar{X}$ coincides with the dividing streamline which leaves the flat plate at the trailing edge. The velocity field $\bar{U}, \bar{V}$ and the displacement functions $\bar{A}_{+}, \bar{A}_{+}$are symmetrical with respect to this streamline. It remains straight and parallel to the plate surface up to the point $\bar{X}_{s}$ where it turns down through an angle which should be chosen in such a way that the reflected shock (that would form in the flow above the wake if its axis remained straight) is cancelled by the expansion fan produced at the turning point. Instead a shock wave is formed at $\bar{X}_{s}$ in the flow below the wake. It generates the pressure jump $\Delta p=R e^{-1 / 4} \bar{P}_{s}$ which is equal to that in the primary shock. As a result the pressure balance (2.26) is kept across the wake both upstream and downstream of point $\bar{X}_{s}$.

From now on we shall consider only the flow above the $\bar{X}$-axis. Using the affine transformations

$$
\begin{array}{lll}
\bar{X}=\frac{\mu_{w}^{-1 / 4} \rho_{w}^{-1 / 2}}{\lambda^{5 / 4} \beta^{3 / 4}} X, & \bar{Y}=\frac{\mu_{w}^{1 / 4} \rho_{w}^{-1 / 2}}{\lambda^{3 / 4} \beta^{1 / 4}} Y, & \bar{U}=\frac{\mu_{w}^{1 / 4} \rho_{w}^{-1 / 2}}{\lambda^{-1 / 4} \beta^{1 / 4}} U, \\
\bar{V}=\frac{\mu_{w}^{3 / 4} \rho_{w}^{-1 / 2}}{\lambda^{-3 / 4} \beta^{-1 / 4}} V, & \bar{A}_{+}=\frac{\mu_{w}^{1 / 4} \rho_{w}^{-1 / 2}}{\lambda^{-1 / 4} \beta^{1 / 4}} A, & \bar{P}=\frac{\mu_{w}^{1 / 2} \rho_{w}^{-1 / 2}}{\lambda^{-1 / 2} \beta^{1 / 2}} P,
\end{array}
$$

where $\beta=\sqrt{M_{\infty}^{2}-1}$, we can represent the interaction problem (2.29)-(2.33) in the following canonical form. The boundary-layer equations are written as

$$
\begin{aligned}
U \frac{\partial U}{\partial X}+V \frac{\partial U}{\partial Y} & =-\frac{\mathrm{d} P}{\mathrm{~d} X}+\frac{\partial^{2} U}{\partial Y^{2}}, \\
\frac{\partial U}{\partial X}+\frac{\partial V}{\partial Y} & =0 .
\end{aligned}
$$

They have to be solved with the no-slip conditions on the upper surface of the plate

$$
U=V=0 \quad \text { at } Y=0, \quad X \leqslant 0,
$$

and the symmetry conditions along the wake centreline

$$
\frac{\partial U}{\partial Y}=V=0 \quad \text { at } \quad Y=0, \quad X>0 .
$$

The initial condition is written as

$$
U=Y \quad \text { at } \quad X=-\infty,
$$

and at the upper edge of the viscous sublayer we have

$$
U \rightarrow Y+A(x)+\cdots \quad \text { as } Y \rightarrow \infty .
$$

The interaction law becomes

$$
P=P_{s} H\left(X-X_{s}\right)-\frac{\mathrm{d} A}{\mathrm{~d} X} .
$$


Here

$$
P_{s}=\frac{\mu_{w}^{-1 / 2} \rho_{w}^{1 / 2}}{\lambda^{1 / 2} \beta^{-1 / 2}} \bar{P}_{s}, \quad X_{s}=\frac{\mu_{w}^{1 / 4} \rho_{w}^{1 / 2}}{\lambda^{-5 / 4} \beta^{-3 / 4}} \bar{X}_{s}
$$

are two similarity parameters controlling the flow in the interaction region.

Finally, we have to take into account that viscous-inviscid interaction allows for upstream influence through the boundary layer even in well-attached flows (see Lighthill 1953). This means that an additional boundary condition specifying the state of the flow downstream of the interaction region is required. We shall assume that

$$
P=P_{s} \quad \text { at } \quad X=\infty .
$$

This condition closes the formulation of the interaction problem (2.34).

\section{Numerical method}

To study the behaviour of the flow in the interaction region, the solution of equations (2.34) was constructed with the help of the so-called direct method which proved to be significantly more stable compared to other numerical techniques used in the the theory of viscous-inviscid interaction. A recent review of these may be found in the monograph by Sychev et al. (1998, chap. 7). The direct method was used earlier to study a number of flows, most notably, the incompressible flow near the trailing edge of a thin aerofoil at an incidence (see Korolev 1989) and, more recently, supersonic flow past a compression ramp (Korolev et al. 2002).

In the version of this method adopted in the present study a uniform grid

$$
\left(X_{j}, Y_{k}\right), \quad\left\{\begin{array}{l}
j=1, \ldots, N, \\
k=1, \ldots, M,
\end{array}\right.
$$

is used to represent the governing equations (2.34) in a finite-difference form. With $j=j_{e}$ assumed to correspond to the trailing edge

$$
X_{j}=\Delta X\left(j-j_{e}\right), \quad Y_{k}=\Delta Y(k-1) .
$$

Here $\Delta X$ and $\Delta Y$ are the mesh steps in $X$ - and $Y$-directions respectively. The grid functions $\left\{U_{j, k}\right\},\left\{V_{j, k}\right\}$ and $\left\{P_{j}\right\}$ are introduced according to the rule

$$
U_{j, k}=U\left(X_{j}, Y_{k}\right), \quad V_{j, k}=V\left(X_{j}, Y_{k}\right), \quad P_{j}=P\left(X_{j}\right) .
$$

\subsection{Finite-differencing of the governing equations}

We shall now describe the manner in which the governing equations (2.34) have been represented in a finite-difference form. For further purposes it is convenient to express the resulting algebraic equations as operator equations

$$
L_{j, k}=0, \quad\left\{\begin{array}{l}
j=1, \ldots, N, \\
k=1, \ldots, M,
\end{array}\right.
$$

where $L_{j, k}$ are the finite-difference operators acting on the grid functions $\left\{U_{j, k}\right\}$ and $\left\{P_{j}\right\}$.

We start with the initial condition (2.34e). Choosing the left-hand-side boundary of the computational domain sufficiently far upstream of the trailing edge, we can write

$$
L_{1, k}=U_{1, k}-\Delta Y(k-1)=0, \quad k=1, \ldots, M .
$$


At the outer edge of the viscous sublayer condition $(2.34 f)$ holds. We write it in the form

$$
\frac{\partial U}{\partial Y}=1 \quad \text { at } \quad Y=\infty
$$

which may be approximated with the second order accuracy as

$$
L_{j, M}=\frac{3 U_{j, M}-4 U_{j, M-1}+U_{j, M-2}}{2 \Delta Y}-1=0, \quad j=2, \ldots, N .
$$

The no-slip condition on the plate surface $(2.34 c)$ and symmetry condition along the wake axis $(2.34 d)$ may be written together in the form

$$
L_{j, 1}=v_{1} U_{j, 1}+v_{2} U_{j, 2}+v_{3} U_{j, 3}=0, \quad j=2, \ldots, N,
$$

where

$$
\left(v_{1}, v_{2}, v_{3}\right)= \begin{cases}(1,0,0) & \text { for } j=2, \ldots, j_{e}, \\ \left(-\frac{3}{2 \Delta Y}, \frac{2}{\Delta Y},-\frac{1}{2 \Delta Y}\right) & \text { for } j=j_{e}+1, \ldots, N .\end{cases}
$$

Now we turn to the internal mesh points where the corresponding operator equations may be obtained by finite-differencing the momentum equation $(2.34 a)$. On the second mesh line $(j=2)$ we use a modification of the Crank \& Nicolson (1947) scheme with windward approximation of the derivative $\partial U / \partial Y$ :

$$
\begin{aligned}
L_{2, k}= & \frac{U_{2, k}+U_{1, k}}{2} \frac{U_{2, k}-U_{1, k}}{\Delta X}+V_{2-\frac{1}{2}, k} \frac{D_{Y}\left\{U_{2, k}\right\}+D_{Y}\left\{U_{1, k}\right\}}{2} \\
& +\frac{P_{2}-P_{1}}{\Delta X}-\frac{D_{Y Y}\left\{U_{2, k}\right\}+D_{Y Y}\left\{U_{1, k}\right\}}{2}=0, \quad k=2, \ldots, M-1 .
\end{aligned}
$$

Here $D_{Y}\left\{U_{j, k}\right\}$ and $D_{Y Y}\left\{U_{j, k}\right\}$ denote the finite-difference representations of the firstand second-order derivatives of $U$ with respect to $Y$ centred at point $(j, k)$. The first-order derivative is calculated as

$$
D_{Y}\left\{U_{j, k}\right\}=\kappa_{1} U_{j, k+2}+\kappa_{2} U_{j, k+1}+\kappa_{3} U_{j, k}+\kappa_{4} U_{j, k-1}+\kappa_{5} U_{j, k-2},
$$

where

$$
\kappa_{1}=\frac{\alpha-1}{4 \Delta Y}, \quad \kappa_{2}=-\frac{\alpha-1}{\Delta Y}, \quad \kappa_{3}=\frac{3 \alpha}{2 \Delta Y}, \quad \kappa_{4}=-\frac{\alpha+1}{\Delta Y}, \quad \kappa_{5}=\frac{\alpha+1}{4 \Delta Y} .
$$

and

$$
\alpha= \begin{cases}\operatorname{sign} V_{2-1 / 2, k} & \text { if } k \neq 2, M-1 \\ -1 & \text { if } \quad k=2 \\ 1 & \text { if } k=M-1\end{cases}
$$

Note that upward and downward approximations are enforced at the lower and upper boundaries of the computational domain. For the second-order derivative a conventional central-difference approximation is used:

$$
D_{Y Y}\left\{U_{j, k}\right\}=\frac{U_{j, k+1}-2 U_{j, k}+U_{j, k-1}}{\Delta Y^{2}} .
$$

Finally, the lateral velocity $V_{2-1 / 2, k}$ is taken in (3.4) halfway between $j=1$ and $j=2$. It may be expressed via $\left\{U_{j, k}\right\}$ by direct integration of the continuity equation $(2.34 b)$ 
with condition $V(X, 0)=0$ :

$$
V_{2-1 / 2, k}=-\frac{1}{2} \Delta Y\left[\frac{U_{2,1}-U_{1,1}}{\Delta X}+2 \sum_{i=2}^{k-1} \frac{U_{2, i}-U_{1, i}}{\Delta X}+\frac{U_{2, k}-U_{1, k}}{\Delta X}\right] .
$$

This allows us to reduce the number of unknowns and to ensure that operators $L_{j, k}$ act upon $\left\{U_{j, k}\right\}$ and $\left\{P_{j}\right\}$ only.

Moving to the mesh lines situated further downstream $(j \geqslant 3)$ we modified the finite-difference representation of the momentum equation $(2.34 a)$ in such a way that it ensures stability of the method if a region of reversed flow $(U<0)$ is encountered. This is achieved by introducing windward approximation for $\partial U / \partial X$. The corresponding finite-difference operator is written as

$$
\begin{aligned}
L_{j, k}=U_{j, k} D_{X}\left\{U_{j, k}\right\}+V_{j, k} D_{Y}\left\{U_{j, k}\right\} & \\
& +\frac{3 P_{j}-4 P_{j-1}+P_{j-2}}{2 \Delta X}-D_{Y Y}\left\{U_{j, k}\right\}=0, \quad k=2, \ldots, M-1,
\end{aligned}
$$

where

$$
D_{X}\left\{U_{j, k}\right\}=\varkappa_{1} U_{j+2, k}+\varkappa_{2} U_{j+1, k}+\varkappa_{3} U_{j, k}+\varkappa_{4} U_{j-1, k}+\varkappa_{5} U_{j-2, k},
$$

with

$$
x_{1}=\frac{\beta-1}{4 \Delta Y}, \quad x_{2}=-\frac{\beta-1}{\Delta Y}, \quad x_{3}=\frac{3 \beta}{2 \Delta Y}, \quad x_{4}=-\frac{\beta+1}{\Delta Y}, \quad x_{5}=\frac{\beta+1}{4 \Delta Y} .
$$

and $\beta=\operatorname{sign} U_{j, k}$. The first- and second-order derivatives of $U$ with respect to $Y$ are given, as before, by formulae (3.5) and (3.6), while for the lateral velocity $V_{j, k}$ is calculated as

$$
V_{j, k}=-\frac{1}{2} \Delta Y\left[D_{x}\left\{U_{j, 1}\right\}+2 \sum_{i=2}^{k-1} D_{x}\left\{U_{j, i}\right\}+D_{x}\left\{U_{j, k}\right\}\right] .
$$

In our calculations finite-difference scheme (3.7) was used for all $j=3, \ldots N$ except on the mesh line $j=j_{e}+1$ situated immediately downstream of the trailing edge. The reason is that the solution of the interaction problem (2.34) develops a singularity at the trailing edge. In particular, we shall see that the pressure gradient appears to be discontinuous at $X=0$. Therefore, to improve the stability of the scheme, the derivatives of $U$ and $P$ with respect to $X$ were approximated by central differences on the mesh line $j=j_{e}+1$ in both the momentum and continuity equations leading to

$$
\begin{aligned}
L_{j_{e}+1, k}=U_{j_{e}+1, k} & \frac{U_{j_{e}+2, k}-U_{j_{e}, k}}{2 \Delta X}+V_{j_{e}+1, k} D_{Y}\left\{U_{j_{e}+1, k}\right\} \\
& +\frac{P_{j_{e}+2}-P_{j_{e}}}{2 \Delta X}-D_{Y Y} D_{Y Y}\left\{U_{j_{e}+1, k}\right\}=0, \quad k=2, \ldots, M-1,
\end{aligned}
$$

where the derivatives of $U$ with respect to $Y$ are given again by (3.6), (3.7) and

$$
V_{j_{e}+1, k}=-\frac{1}{2} \Delta Y\left[\frac{U_{j_{e}+2,1}-U_{j_{e}, 1}}{2 \Delta X}+2 \sum_{i=2}^{k-1} \frac{U_{j_{e}+2, i}-U_{j_{e}, i}}{2 \Delta X}+\frac{U_{j_{e}+2, k}-U_{j_{e}, k}}{2 \Delta X}\right] .
$$

It remains to describe the finite-differencing of the interaction law. In order to exclude the displacement function $A(X)$ from the set of unknowns, we combine 
$(2.34 f)$ and $(2.34 g)$ together leading to

$$
P=P_{s} H\left(X-X_{s}\right)-\left.\frac{\partial U}{\partial X}\right|_{Y \rightarrow \infty} .
$$

When representing this equation in finite differences one has to keep in mind that it is due to the interaction that the upstream influence in the boundary layer is observed. Hence, in order to ensure that numerical solution of the interaction problem (2.34) shows dependence on the state of the flow (2.34h) downstream of the trailing edge, we express the interaction law (3.9) as

$$
\begin{aligned}
& L_{j, M+1}=\frac{P_{j+1}+P_{j}}{2}-P_{s} \frac{H\left(X_{j+1}-X_{s}\right)+H\left(X_{j}-X_{s}\right)}{2} \\
&+\frac{U_{j+1, M}-U_{j, M}}{\Delta X}=0, \quad j=1, \ldots N-1 .
\end{aligned}
$$

To close the formulation of the finite-difference equations, the downstream condition $(2.34 h)$ is written in the following operator form:

$$
L_{N, M+1}=P_{N}-P_{s}=0 .
$$

\subsection{Matrix Thomas technique}

In order to employ the matrix Thomas technique we express the finite-difference equations in a vector form. For this purpose we introduce a set of vector operators $\mathbf{L}_{j}$, each being composed of the scalar finite-difference operators $L_{k, j}, k=1, \ldots, M+1$ acting on the grid line $X=X_{j}$. We also arrange the unknown quantities into vectors $\boldsymbol{\Omega}_{j}$ by combining together the elements of the velocity grid function $U_{k, j}$ on the $j$ th grid line with pressure $P_{j}$. We have

$$
\mathbf{L}_{j}=\left[\begin{array}{c}
L_{j, 1} \\
\vdots \\
L_{j, k} \\
\vdots \\
L_{j, M} \\
L_{j, M+1}
\end{array}\right], \quad \boldsymbol{\Omega}_{j}=\left[\begin{array}{c}
U_{j, 1} \\
\vdots \\
U_{j, k} \\
\vdots \\
U_{j, M} \\
P_{j}
\end{array}\right], \quad j=1, \ldots, N
$$

We shall also introduce a vector $\boldsymbol{C}$ of governing parameters which may include the shock strength $P_{s}$, its position $X_{s}$, etc. The precise composition of this vector is not important at this stage; we can continue describing the method without defining the elements of $\boldsymbol{C}$ explicitly.

Using the vector notation one can combine equations (3.1) with the first $(j=1)$ of equations (3.10) and write them together in the more compact form

$$
\mathbf{L}_{1}\left(\boldsymbol{\Omega}_{1}, \boldsymbol{\Omega}_{2}, \boldsymbol{C}\right)=0 .
$$

Similarly, combining the first elements $(j=2)$ of the boundary conditions (3.2) and (3.3) with the set of equations (3.4) and the second $(j=2)$ of equations (3.10) results in

$$
\mathbf{L}_{2}\left(\boldsymbol{\Omega}_{1}, \boldsymbol{\Omega}_{2}, \boldsymbol{\Omega}_{3}, \boldsymbol{C}\right)=0 .
$$

The vector equations for the subsequent grid lines

$$
\mathbf{L}_{j}\left(\boldsymbol{\Omega}_{j-2}, \boldsymbol{\Omega}_{j-1}, \boldsymbol{\Omega}_{j}, \boldsymbol{\Omega}_{j+1}, \boldsymbol{\Omega}_{j+2}, \boldsymbol{C}\right)=0, \quad j=3, \ldots, N-2,
$$


are composed of the corresponding elements of equations (3.7) and (3.8), boundary conditions (3.2), (3.3) and equation (3.10). The dependence of $\mathbf{L}_{j}$ in (3.14) on $\boldsymbol{\Omega}_{j+1}$ and $\boldsymbol{\Omega}_{j+2}$ comes, first of all, from equation (3.7) where the windward finite-difference operator $D_{x}\left\{U_{j, k}\right\}$ is used for representing derivative $\partial U / \partial X$ in equation (2.34a). For all the node points with negative $U_{j, k}$ this operator is calculated using $U_{j+1, k}$ and $U_{j+2, k}$. However, when calculating the flow one has to choose the computational domain such that its right-hand-side boundary is taken far enough downstream of any region of flow reversal. This means that near the right-hand-side boundary equation does not really involve $U_{j+1, k}$ and $U_{j+2, k}$. The vector operator $\mathbf{L}_{j}$, nevertheless, remains dependent on $\boldsymbol{\Omega}_{j+1}$ due to the interaction equation (3.10). This means that the equation corresponding to $j=N-1$ may be written as

$$
\mathbf{L}_{N-1}\left(\boldsymbol{\Omega}_{N-3}, \boldsymbol{\Omega}_{N-2}, \boldsymbol{\Omega}_{N-1}, \boldsymbol{\Omega}_{N}, \boldsymbol{C}\right)=0 .
$$

On the last grid line $(j=N)$ we use the downstream boundary condition (3.11) instead of the interaction law (3.10), and therefore the corresponding operator equation is written as

$$
\mathbf{L}_{N}\left(\boldsymbol{\Omega}_{N-2}, \boldsymbol{\Omega}_{N-1}, \boldsymbol{\Omega}_{N}, \boldsymbol{C}\right)=0 .
$$

To solve (3.12)-(3.16) Newton-Raphson linearization has been used. With $\hat{\boldsymbol{\Omega}}_{j}, \hat{\boldsymbol{C}}$ denoting an approximation to the solution given as an initial guess or found from the previous iteration, an improved approximation

$$
\boldsymbol{\Omega}_{j}=\hat{\boldsymbol{\Omega}}_{j}+\Delta \boldsymbol{\Omega}_{j}, \quad \boldsymbol{C}=\hat{\boldsymbol{C}}+\Delta \boldsymbol{C}
$$

may be found by substituting (3.17) into (3.12)-(3.16) and making use of the Taylor expansions. This results in the following linear equations:

$$
\begin{gathered}
\frac{\partial \mathbf{L}_{1}}{\partial \boldsymbol{\Omega}_{1}} \Delta \boldsymbol{\Omega}_{1}+\frac{\partial \mathbf{L}_{1}}{\partial \boldsymbol{\Omega}_{2}} \Delta \boldsymbol{\Omega}_{2}+\frac{\partial \mathbf{L}_{1}}{\partial \boldsymbol{C}} \Delta \boldsymbol{C}=-\mathbf{L}_{1}, \\
\frac{\partial \mathbf{L}_{2}}{\partial \boldsymbol{\Omega}_{1}} \Delta \boldsymbol{\Omega}_{1}+\frac{\partial \mathbf{L}_{2}}{\partial \boldsymbol{\Omega}_{2}} \Delta \boldsymbol{\Omega}_{2}+\frac{\partial \mathbf{L}_{2}}{\partial \boldsymbol{\Omega}_{3}} \Delta \boldsymbol{\Omega}_{3}+\frac{\partial \mathbf{L}_{2}}{\partial \boldsymbol{C}} \Delta \boldsymbol{C}=-\mathbf{L}_{2}, \\
\frac{\partial \mathbf{L}_{j}}{\partial \boldsymbol{\Omega}_{j-2}} \Delta \boldsymbol{\Omega}_{j-2}+\frac{\partial \mathbf{L}_{j}}{\partial \boldsymbol{\Omega}_{j-1}} \Delta \boldsymbol{\Omega}_{j-1}+\frac{\partial \mathbf{L}_{j}}{\partial \boldsymbol{\Omega}_{j}} \Delta \boldsymbol{\Omega}_{j}+\frac{\partial \mathbf{L}_{j}}{\partial \boldsymbol{\Omega}_{j+1}} \Delta \boldsymbol{\Omega}_{j+1} \\
+\frac{\partial \mathbf{L}_{j}}{\partial \boldsymbol{\Omega}_{j+2}} \Delta \boldsymbol{\Omega}_{j+2}+\frac{\partial \mathbf{L}_{j}}{\partial \boldsymbol{C}} \Delta \boldsymbol{C}=-\mathbf{L}_{j}, \quad j=3, \ldots, N-2, \\
\frac{\partial \mathbf{L}_{N-1}}{\partial \boldsymbol{\Omega}_{N-3}} \Delta \boldsymbol{\Omega}_{N-3}+\frac{\partial \mathbf{L}_{N-1}}{\partial \boldsymbol{\Omega}_{N-2}} \Delta \boldsymbol{\Omega}_{N-2}+\frac{\partial \mathbf{L}_{N-1}}{\partial \boldsymbol{\Omega}_{N-1}} \Delta \boldsymbol{\Omega}_{N-1} \\
+\frac{\partial \mathbf{L}_{N-1}}{\partial \boldsymbol{\Omega}_{N}} \Delta \boldsymbol{\Omega}_{N}+\frac{\partial \mathbf{L}_{N-1}}{\partial \boldsymbol{C}} \Delta \boldsymbol{C}=-\mathbf{L}_{N-1}, \\
\frac{\partial \mathbf{L}_{N}}{\partial \boldsymbol{\Omega}_{N-2}} \Delta \boldsymbol{\Omega}_{N-2}+\frac{\partial \mathbf{L}_{N}}{\partial \boldsymbol{\Omega}_{N-1}} \Delta \boldsymbol{\Omega}_{N-1}+\frac{\partial \mathbf{L}_{N}}{\partial \boldsymbol{\Omega}_{N}} \Delta \boldsymbol{\Omega}_{N}+\frac{\partial \mathbf{L}_{N}}{\partial \boldsymbol{C}} \Delta \boldsymbol{C}=-\mathbf{L}_{N} .
\end{gathered}
$$

For this study two versions of a computer program based on matrix extension of Thomas elimination algorithm were written. In the first one all the governing parameters were treated as given, which is equivalent to setting $\boldsymbol{C}=0$ in (3.17) and disregarding the corresponding terms in (3.18)-(3.22). These equations were then solved based on the recurrent use of the formula

$$
\Delta \boldsymbol{\Omega}_{j}=\mathscr{R}_{j} \Delta \boldsymbol{\Omega}_{j+1}+\mathscr{T}_{j} \Delta \boldsymbol{\Omega}_{j+2}+\boldsymbol{S}_{j}, \quad j=1, \ldots, N-2 .
$$


Here $\mathscr{R}_{j}$ and $\mathscr{T}_{j}$ are $(M+1) \times(M+1)$ matrices, and $\boldsymbol{S}_{j}$ an $(M+1)$-component vectors. For $j=N-1$ and $j=N$ formula (3.23) reduces to

$$
\Delta \boldsymbol{\Omega}_{N-1}=\mathscr{R}_{N-1} \Delta \boldsymbol{\Omega}_{N}+\boldsymbol{S}_{N-1}, \quad \Delta \boldsymbol{\Omega}_{N}=\boldsymbol{S}_{N} .
$$

The calculations were perform by choosing a certain position $X_{s}$ of the impinging shock and gradually increasing the shock strength $P_{s}$. Using this approach we were able to construct the solution of the interaction problem for a wide range of variation of $P_{s}$ for all $X_{s}<3.2$. At the same time we found that for $X_{s}>3.2$ the Newtonian iterations converged reasonably well only up to a certain critical value $P_{s}^{c}$ of shock strength $P_{s}$ which was found to be dependent on $X_{s}$; beyond $P_{s}^{c}$ no convergence could be achieved.

This result came as no surprise to us. Given that $X_{s}$ is large enough one can expect two (or more) states of the flow for the same shock strength $P_{s}$. Indeed, since the velocity in the wake grows with the distance $X_{s}$ downstream of the trailing edge, the pressure rise that is large enough to cause local flow reversal in the wake at the position $X_{s}$, can also cause a formation of an extended region flow reversal that starts at an upstream location in the wake or even in the boundary layer on the plate surface.

To study possible non-uniqueness of the solution a second version of the program was written. This time the shock strength $P_{s}$ was treated as an unknown quantity. It was included as the first element in the governing parameter vector

$$
\boldsymbol{C}=\left[\begin{array}{c}
P_{s} \\
U_{s}
\end{array}\right]
$$

with the second element $U_{s}$ being the velocity on the wake axis at the shock location. Assuming that the shock impinges on the wake at a position where $j=j_{s}$ one can write

$$
U_{s}=U_{j_{s}, 1} .
$$

With this approach the full set of equations (3.18)-(3.22) should be considered. Their solution may be written in the form

$$
\Delta \boldsymbol{\Omega}_{j}=\mathscr{R}_{j} \Delta \boldsymbol{\Omega}_{j+1}+\mathscr{T}_{j} \Delta \boldsymbol{\Omega}_{j+2}+\mathscr{Z}_{j} \Delta \boldsymbol{C}+\boldsymbol{S}_{j}, \quad j=1, \ldots, N-2,
$$

which represents an obvious generalization of recurrent formulae (3.23). An additional Thomas coefficient $\mathscr{Z}_{j}$ is an $(M+1) \times 2$ matrix. For $j=N-1$ and $j=N$ formula (3.26) reduces to

$$
\left.\begin{array}{rl}
\Delta \boldsymbol{\Omega}_{N-1} & =\mathscr{R}_{N-1} \Delta \boldsymbol{\Omega}_{N}+\mathscr{Z}_{N-1} \Delta \boldsymbol{C}+\boldsymbol{S}_{N-1}, \\
\Delta \boldsymbol{\Omega}_{N} & =\mathscr{Z}_{N} \Delta \boldsymbol{C}+\boldsymbol{S}_{N} .
\end{array}\right\}
$$

Recursive formulae to calculate the Thomas coefficients $\mathscr{R}_{j}, \mathscr{T}_{j}, \mathscr{Z}_{j}$ and $\boldsymbol{S}_{j}$ may be deduced in a usual way by substituting (3.26) and (3.27) into equations (3.18)-(3.22). With known Thomas coefficients formulae (3.27), (3.27) may be rearranged as

$$
\Delta \boldsymbol{\Omega}_{j}=\mathscr{F}_{j} \Delta \boldsymbol{C}+\boldsymbol{G}_{j}, \quad j=1, \ldots, N,
$$

where matrices $\mathscr{F}_{j}$ and vectors $\boldsymbol{G}_{j}$ are calculated recursively using

$$
\left.\begin{array}{rl}
\mathscr{F}_{j} & =\mathscr{Z}_{j}+\mathscr{R}_{j} \mathscr{F}_{j+1}+\mathscr{T}_{j} \mathscr{F}_{j+2}, \\
\boldsymbol{G}_{j} & =\boldsymbol{S}_{j}+\mathscr{R}_{j} \boldsymbol{G}_{j+1}+\mathscr{T}_{j} \boldsymbol{G}_{j+2},
\end{array}\right\} \quad j=1, \ldots, N-2,
$$


and from (3.27)

$$
\begin{array}{cc}
\mathscr{F}_{N}=\mathscr{Z}_{N}, & \mathscr{\mathscr { F }}_{N-1}=\mathscr{R}_{N-1} \mathscr{F}_{N}+\mathscr{Z}_{N-1}, \\
\boldsymbol{G}_{N}=\boldsymbol{S}_{N}, & \boldsymbol{G}_{N-1}=\mathscr{R}_{N-1} \boldsymbol{G}_{N}+\boldsymbol{S}_{N-1} .
\end{array}
$$

To determine the unknown vector $\boldsymbol{C}$ we need to formulate two additional operator equations. The first follows directly from (3.25):

$$
Q_{1}=U_{j_{s}, 1}-U_{s}=0 \text {. }
$$

The second depends on the way the calculations are organized. In the present study we marched along a hysteresis curve in the $\left(P_{s}, U_{s}\right)$-plane (see, for example, figure 5) in steps corresponding to a constant arclength, $\Delta S=0.05$. If $\bar{P}_{s}, \bar{U}_{s}$ represent a point on a hysteresis curve then for the next point $\left(P_{s}, U_{s}\right)$ we have

$$
Q_{2}=\left(P_{s}-\bar{P}_{s}\right)^{2}+\left(U_{s}-\bar{U}_{s}\right)^{2}-\Delta S^{2}=0 .
$$

Equations (3.29), (3.30) may be combined into a vector equation

$$
\mathbf{Q}\left(\boldsymbol{\Omega}_{1}, \ldots, \boldsymbol{\Omega}_{j}, \ldots, \boldsymbol{\Omega}_{N}, \boldsymbol{C}\right)=0,
$$

where $\mathbf{Q}$ is a two-component vector operator. This equation is linearized in a usual way leading to

$$
\sum_{j=1}^{N} \frac{\partial \mathbf{Q}}{\partial \boldsymbol{\Omega}_{j}} \Delta \boldsymbol{\Omega}_{j}+\frac{\partial \mathbf{Q}}{\partial \boldsymbol{C}} \Delta \boldsymbol{C}+\mathbf{Q}=0 .
$$

Combining (3.31) with (3.28) we have the following equation:

$$
\left(\sum_{j=1}^{N} \frac{\partial \mathbf{Q}}{\partial \boldsymbol{\Omega}_{j}} \mathscr{F}_{j}+\frac{\partial \mathbf{Q}}{\partial \boldsymbol{C}}\right) \Delta \boldsymbol{C}+\sum_{j=1}^{N} \frac{\partial \mathbf{Q}}{\partial \boldsymbol{\Omega}_{j}} \boldsymbol{G}_{j}+\mathbf{Q}=0,
$$

which should be solved for $\Delta \boldsymbol{C}$. Then the correction vectors $\Delta \boldsymbol{\Omega}_{j}$ may be calculated by making use of formula (3.28). The solution is then updated using (3.17), and the iterations are repeated until the convergence is reached.

\subsection{Marching scheme}

For comparison purposes we also reconstructed the marching method used by a number of researchers in early calculations of supersonic flows with viscous-inviscid interaction. In particular, for the trailing-edge flow it was applied by Daniels (1974). The method is based on the observation that the solution of the interaction problem (2.34) may be represented far upstream of the trailing edge $(X \rightarrow-\infty)$ in the following asymptotic form:

$$
P=B \mathrm{e}^{\lambda_{0} X}, \quad U=Y+B \mathrm{e}^{\lambda_{0} X} \frac{\lambda_{0}^{1 / 3}}{\operatorname{Ai}^{\prime}(0)} \int_{0}^{\lambda_{0}^{1 / 3} Y} \operatorname{Ai}(z) \mathrm{d} z .
$$

Here $\operatorname{Ai}(z)$ is the Airy function, $\lambda_{0}$ a universal positive constant related to the derivative of the Airy function at zero value of the argument:

$$
\lambda_{0}=\left[-3 \mathrm{Ai}^{\prime}(0)\right]^{3 / 4} \text {. }
$$

Finally, factor $B$ represents the amplitude of perturbations produced in the flow by upstream propagating disturbances.

The calculations were performed based on the vorticity equation

$$
U \frac{\partial \omega}{\partial X}+V \frac{\partial \omega}{\partial Y}=\frac{\partial^{2} \omega}{\partial Y^{2}}
$$




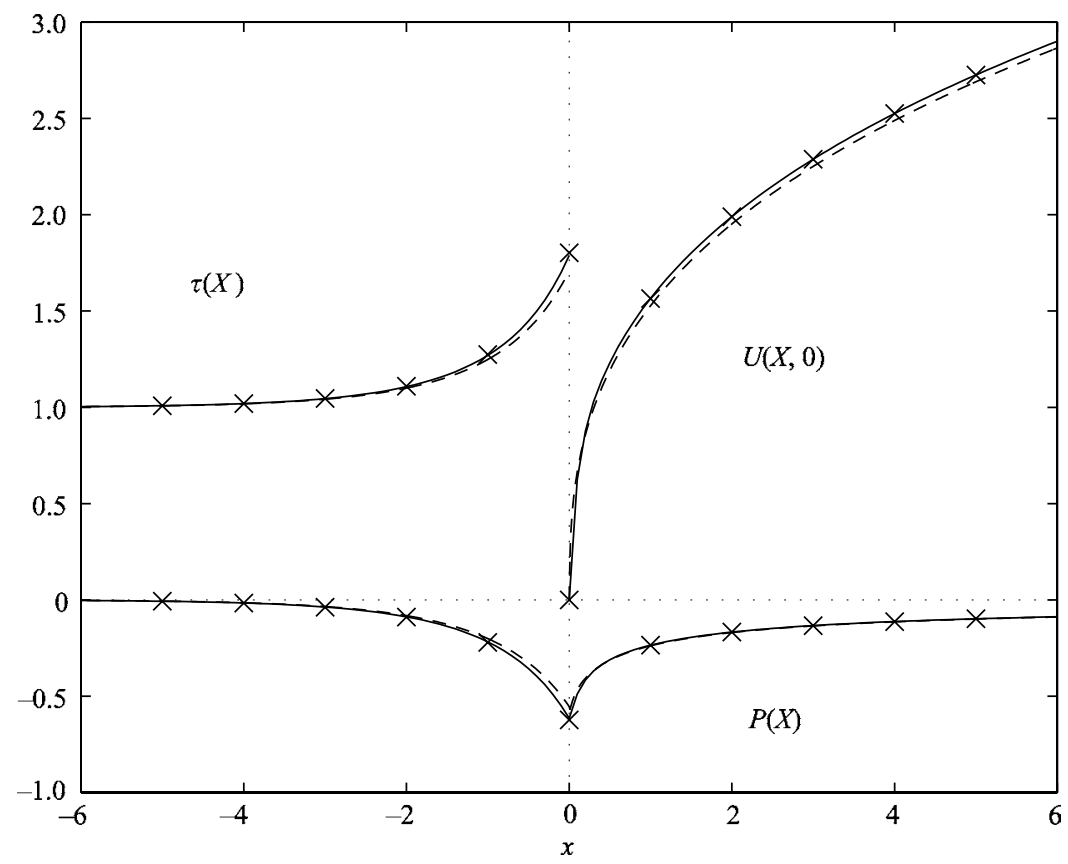

Figure 3. Comparison of Daniels' (1974) data $(\times)$, marching code (dashed) and direct matrix solution (solid) for $P_{s}=0$.

which may be easily deduced by differentiating the momentum equation $(2.34 a)$ with respect to $Y$. We used three-point-backward approximation for $\partial \omega / \partial X$ in this equation for mesh point with $U>0$ and Reyhner \& Flügge-Lotz (1968) approximation in the region of flow reversal $U<0$. Windward difference, similar to (3.5) was used for $\partial \omega / \partial Y$, and the viscous term $\partial^{2} \omega / \partial Y^{2}$ was approximated by the central difference (3.6).

In the marching scheme the calculations are performed successively, moving from one mesh line $X_{j}$ to the next $X_{j+1}$ situated further downstream. The procedure involves two iteration processes, local and global. The former is used on each mesh line $X_{j}$ to update the velocity components $U, V$, and to adjust the induced pressure to the displacement thickness of the boundary layer. The latter is aimed at determining the perturbation amplitude parameter $B$ in (3.32) in such a way that the downstream boundary condition $(2.34 h)$ is satisfied. Newtonian iterations were used for this purpose in our calculations.

Similar to the first method this scheme provides second order accuracy with respect to both $x$ - and $y$-coordinates.

\section{Computational results}

The numerical schemes described above were tested on the previously studied problem of the trailing edge flow with no shock wave, which corresponds to $P_{s}=0$. Daniels (1974) looked at this problem using a multi-grid method based on the stream function formulation of the interaction problem $(2.34 a)$. Figure 3 shows a comparison between his results and data we obtained for a uniform mesh using both the direct matrix and marching schemes. We used a mesh of size of $\Delta X=0.01, \Delta Y=0.05$ in the 


\begin{tabular}{lll}
\hline & $P(0)$ & $\tau(0)$ \\
Direct & -0.61473 & 1.78870 \\
Marching & -0.55383 & 1.70716 \\
Daniels & -0.6230 & 1.8026 \\
TABLE 1. Trailing-edge results for $P_{s}=0$. \\
\hline
\end{tabular}

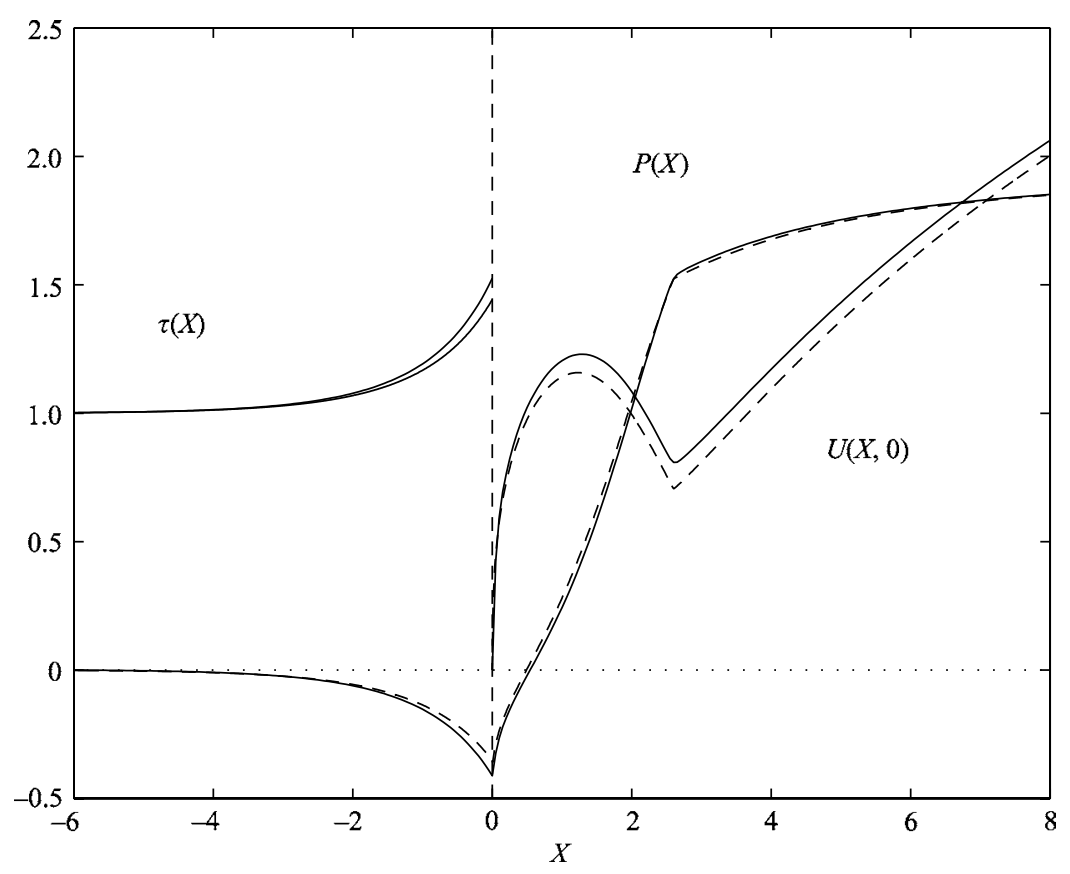

Figure 4. Comparison of direct matrix (solid) and marching (dashed) solutions for $X_{s}=2.6$, $P_{s}=2.0$.

marching scheme and $\Delta X=\Delta Y=0.1$ for the direct matrix calculation. The pressure and skin friction, defined as

$$
\tau(X)=\left.\frac{\partial U}{\partial Y}\right|_{Y=0},
$$

at the trailing edge are shown in table 1.

We see that there is a good agreement with Daniels' results, especially for the direct matrix code. The marching scheme was found to be less accurate which, in our view, may be explained by apparent weakness of shooting based on the exponentially small term in the upstream asymptote (3.32). The problem is highlighted in a second comparison drawn between the two numerical schemes. Figure 4 shows the skin friction $\tau$, pressure $P$ and streamwise velocity in the wake axis $U(X, 0)$ as found by the two numerical schemes. In the matrix solution we used a mesh $\Delta X=0.05, \Delta Y=0.05$. No noticeable change was seen when this mesh was reduced to $\Delta X=\Delta Y=0.1$. At the same time a mesh independent solution was found using the marching code only when a much finer mesh was used, $\Delta X=0.005, \Delta Y=0.05$. Both solutions were calculated in the $(X, Y)$ domain $[-10,15] \times[0,10]$ assuming that $X_{s}=2.6$ and $P_{s}=2.0$. 


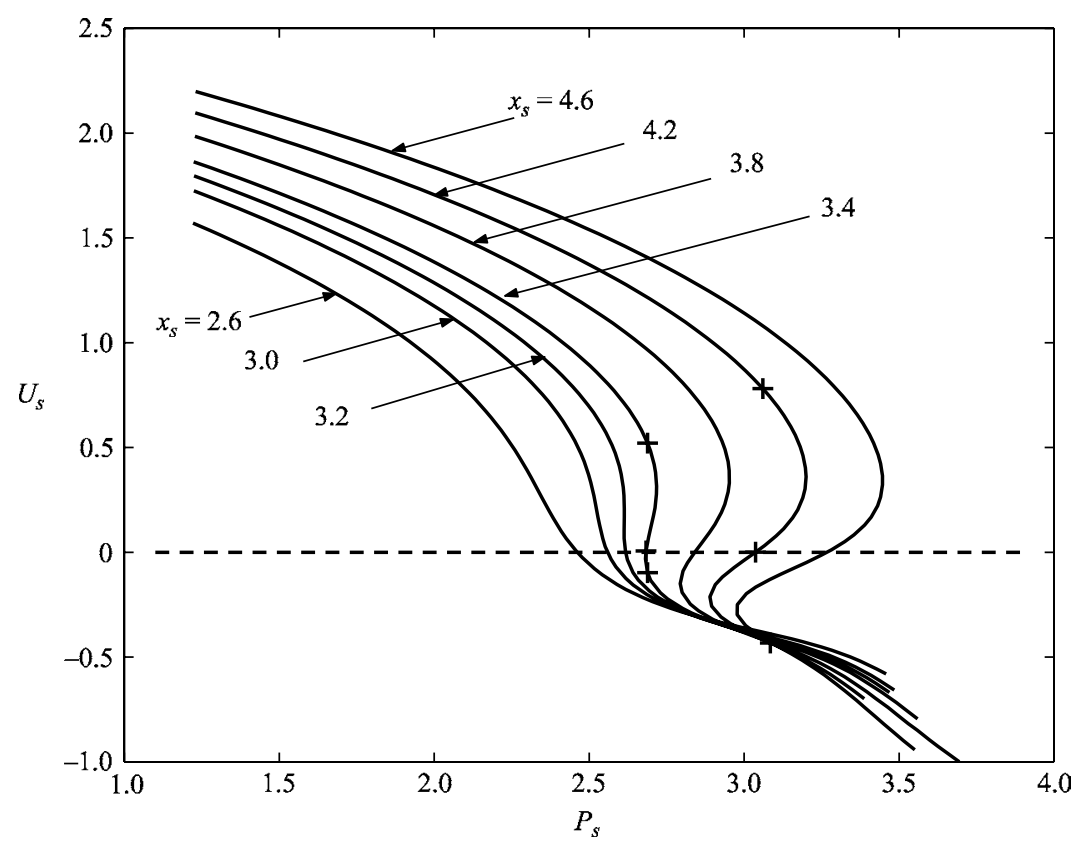

FIGURE 5. Hysteresis plots for shock positions from $X_{s}=2.6$ to $X_{s}=4.6$.

As a consequence of the inability of the marching scheme to model reverse flow regions accurately the direct matrix code has been used to produce the remaining results. The behaviour of the solution, as the shock moves downstream, is shown in the hysteresis diagram (figure 5). It shows that the solution bifurcates when $X_{s}$ reaches a critical value close to 3.2. For all $X_{s}>3.2$ the interaction problem (2.34) admits three solutions. These were calculated using a mesh in which $\Delta X=\Delta Y=0.2$ and $(X, Y) \in[-5,15] \times[0,15]$.

To ensure that the results were mesh independent, we repeated the calculations for $X_{s}=4.6$ with four different computational domains. These are marked in figure 6 as ' + ' for $(X, Y) \in[-5,15] \times[0,15]$, 'o' for $(X, Y) \in[-10,15] \times[0,15]$ and ' $\times$ ' for $(X, Y) \in[-5,15] \times[0,20]$; the mesh steps in these calculations were $\Delta X=\Delta Y=0.2$. A second comparison was made by varying the mesh steps. For a shock location $X_{s}=3.8$, and using the computational domain $[-5,15] \times[0,15]$, the solution for $\Delta X=\Delta Y=0.2$ is marked in figure 6 with ' $\diamond$ ' and that for $\Delta X=0.1, \Delta Y=$ 0.15 with ' $\square$ '. In each case close agreement between the hysteresis curves can be observed.

Typical behaviour of the solutions on the three branches is shown in figure 7 for two sets of the governing parameters: $X_{s}=3.4, P_{s} \approx 2.69$ and $X_{s}=4.2, P_{s} \approx 3.06$. Notice that negative skin friction on the plate surface $(X<0)$ and/or negative velocity on the wake axis $(X>0)$ manifest a formation of reversed flow regions. These are clearly seen in figure 8 , where the streamline patterns are shown for the flow regimes of figure 7. In both cases the flow on the lower branch contains an extended separation region, whilst on the upper branch, no separation is observed.

An important conclusion that may be drawn from the calculation results is that if we imagine an experiment in which the shock position were kept fixed and above $X_{s}=3.2$ and the pressure were increased monotonically we would see the sudden 


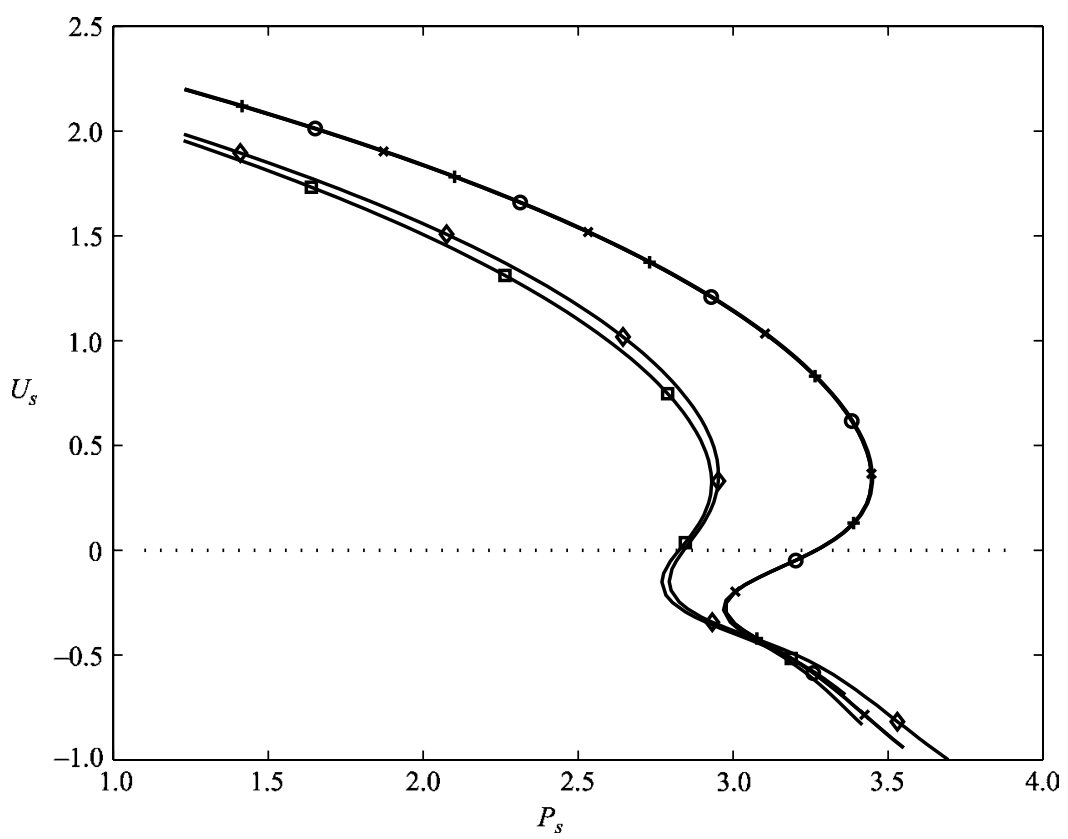

FIGURE 6. Comparison plots examining domain and mesh dependence.

formation of a large separation bubble as the pressure $P_{s}$ reaches its critical value (see figures 5 and 6) and the solution 'jumps' from the upper to lower branch.

\section{Inviscid-inviscid interaction}

We shall assume now that the shock impinges upon the wake downstream of the viscous-inviscid interaction region that occupies an $O\left(R e^{-3 / 8}\right)$ vicinity of the trailing edge. The analysis that follows reveals that in this case the deceleration of the flow in the wake is predominantly inviscid. The viscosity becomes important only in a smaller vicinity of the point of flow reversal $(\S 6)$. This explains why inviscid theoretical models by Mahesh (1996) and Smart \& Kalkhoran (1997) proved to give a good approximation to the vortex breakdown criterion.

To simplify the analysis we shall also assume that the distance $l_{s}$ between the trailing edge and the point of shock-wake interaction (shown as point $S$ in figure 1) is still relatively small as compared to the plate length. This restriction allows us, as we shall see, to treat the pressure in the shock-wake interaction region as being independent of the transverse coordinate measured across the wake. The assumptions made may be expressed as

$$
1 \gg l_{s} \gg R e^{-3 / 8} \text {. }
$$

\subsection{Near-wake solution}

Before analysing the process of interaction of the shock wave with the wake we need to know the behaviour of the flow approaching the interaction region. The latter is expected to occupy a small vicinity of point $S$ (see figure 1 ). For the flow in the wake between the trailing edge and interaction region the boundary-layer equations (2.2) are valid. The boundary conditions at the outer edge of the boundary layer (2.4) also hold. However, the no-slip conditions (2.5) on the plate surface should now be 

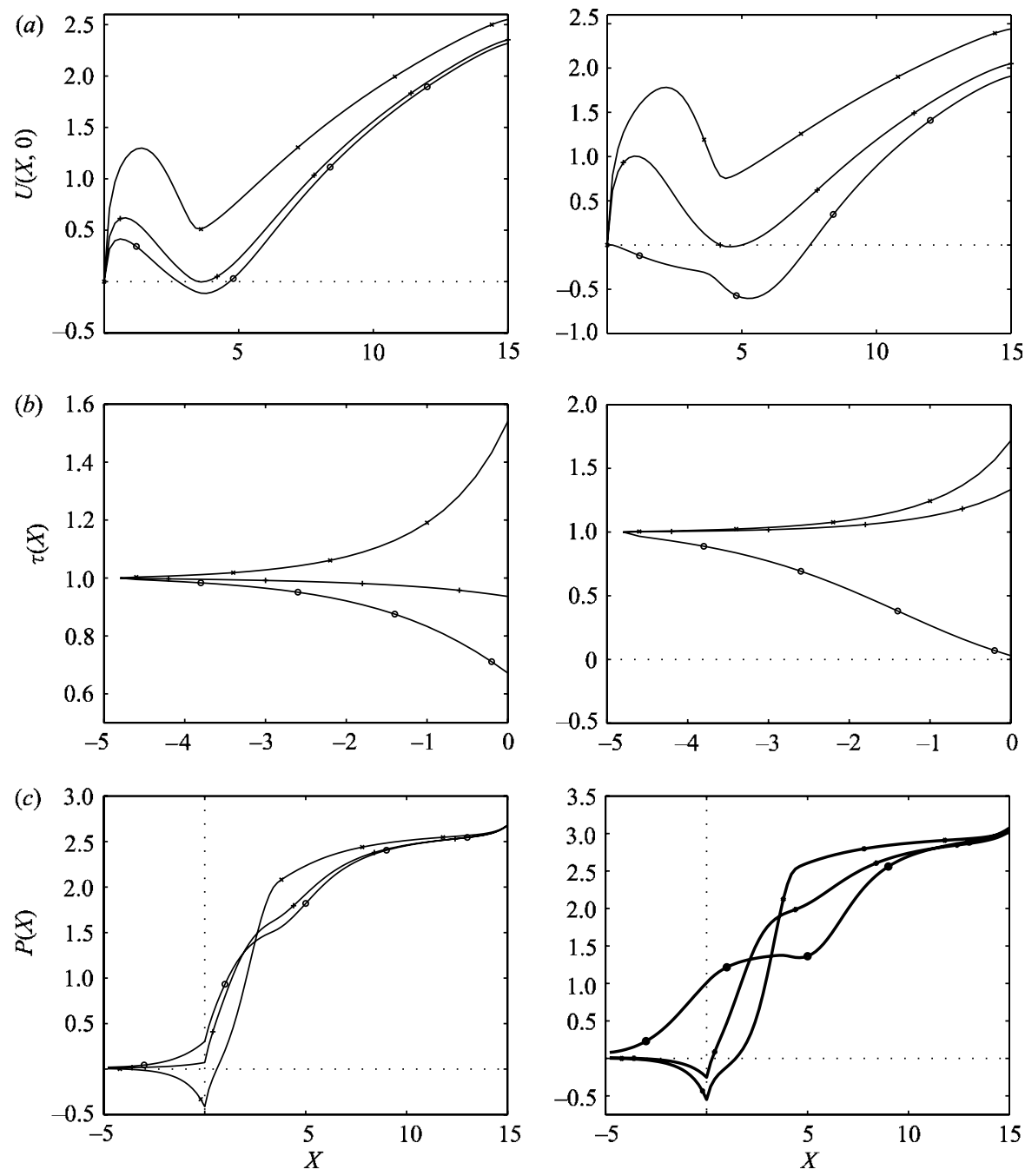

FIgURE 7. Comparison of the three solution branches at $X_{s}=3.4, P_{s} \approx 2.69$ (left) and $X_{s}=4.2, P_{s} \approx 3.06$ (right), where $\times$ is upper branch, + middle branch, $\bigcirc$ lower branch. $(a)$ Wake axis velocity, $U(X, 0) ;(b)$ skin friction, $\tau(X) ;(c)$ pressure, $P(X)$.

substituted by the symmetry conditions on the wake axis

$$
V_{0}=\frac{\partial U_{0}}{\partial Y}=\frac{\partial h_{0}}{\partial Y}=0 \quad \text { at } \quad Y=0 .
$$

The solution of the boundary-layer equations close to the trailing edge is given by the Goldstein (1930) 'near-wake' theory; see also Sychev et al. (1998), chap. 3. Goldstein demonstrated that when the flat plate boundary-layer is continued through the trailing edge into the wake, a two-tiered structure (shown in figure 9) should be considered.

In region $A$ which occupies the main part of the wake, asymptotic analysis of the boundary-layer equations (2.2) is based on the limit

$$
Y=O(1), \quad s=x-1 \rightarrow 0^{+} .
$$




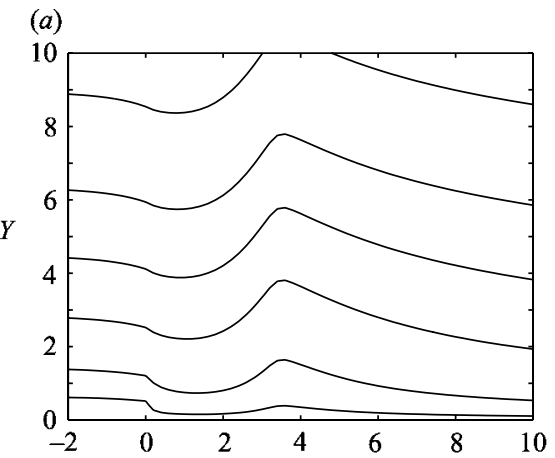

(c)
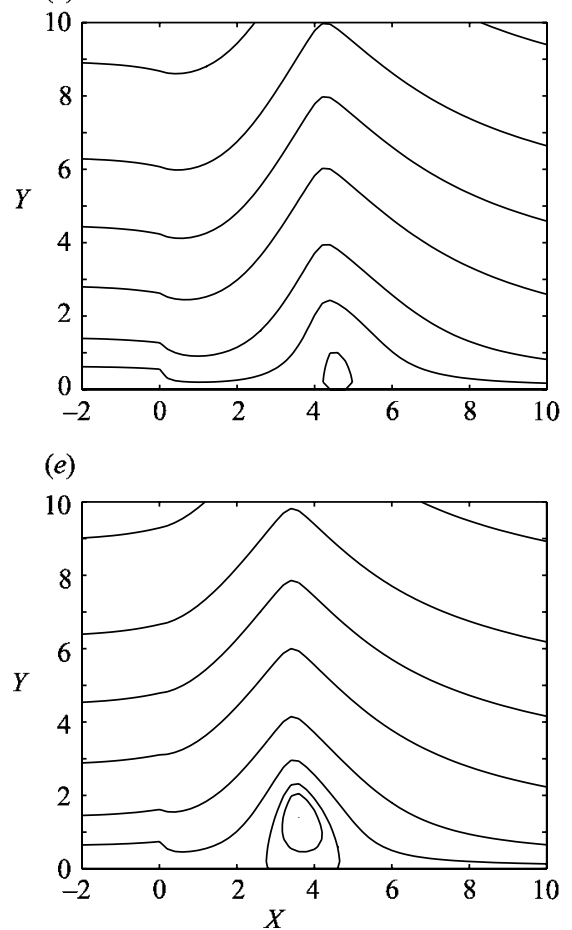

(b)

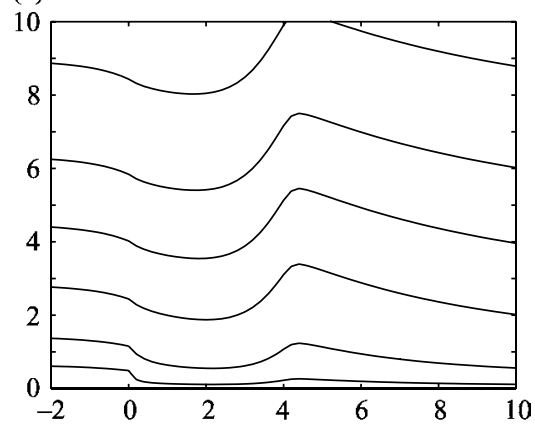

(d)

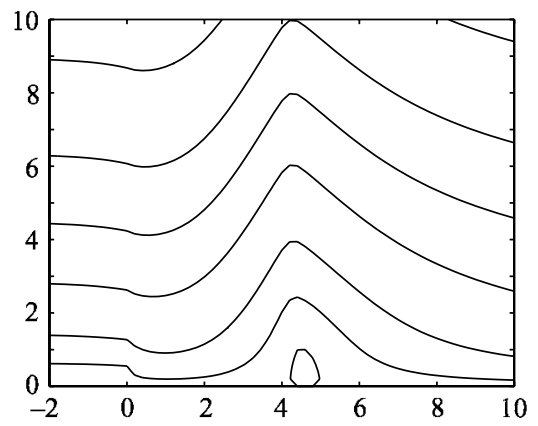

$(f)$

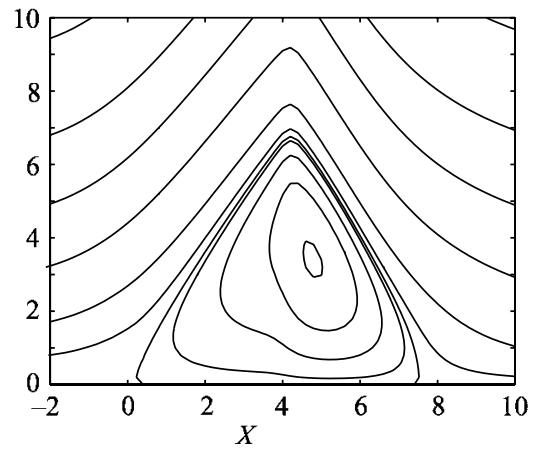

FIgURE 8. Streamline plots for $X_{s}=3.4$ (left) and $X_{s}=4.2$ (right) on the three branches. (a) Upper branch, $P_{s}=2.688$; $(b)$ upper branch, $P s=3.061 ;(c)$ mid-branch, $P_{s}=2.684$; $(d)$ mid-branch, $P_{s}=3.037$; $(e)$ lower branch, $P_{s}=2.688$; $(f)$ lower-branch, $P_{s}=3.084$.

Since the solution is expected to be continuous, one can express the leading order term for $U_{0}, h_{0}, \rho_{0}$ and $\mu_{0}$ in region $A$ in the form

$$
\left.\begin{array}{ll}
U_{0}(x, Y)=U_{00}(Y)+\cdots, & h_{0}(x, Y)=h_{00}(Y)+\cdots, \\
\rho_{0}(x, Y)=\rho_{00}(Y)+\cdots, & \mu_{0}(x, Y)=\mu_{00}(Y)+\cdots .
\end{array}\right\}
$$

Functions $U_{00}(Y), h_{00}(Y), \rho_{00}(Y)$ and $\mu_{00}(Y)$ coincide with those in (2.7), and therefore may be represented at small values of $Y$ by formulae (2.8). Of particular interest here is the behaviour of the longitudinal velocity component

$$
U_{00}(Y)=\lambda Y+\cdots \quad \text { as } \quad Y \rightarrow 0 .
$$




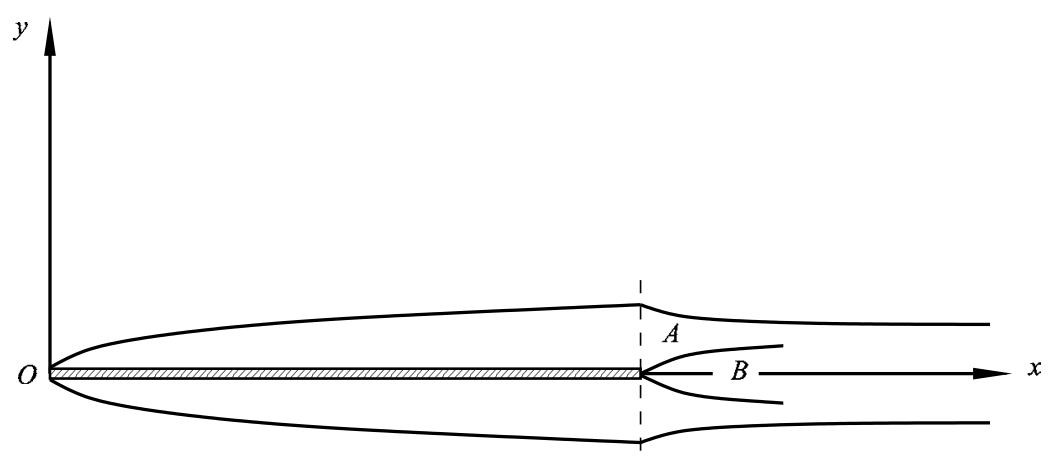

FIGURE 9. Two-tiered structure of the near-wake flow.

It may be easily seen from (5.3) and (5.4) that the solution in region $A$ does not satisfy the symmetry condition (5.2) for the derivative of $U_{0}$. This is why one has to introduce an additional region $B$ situated near the wake axis.

When describing the flow in region $B$ it is convenient to use the stream function $\Psi_{0}(x, Y)$. Based on the continuity equation $(2.2 c)$ we define $\Psi_{0}(x, Y)$ by the equations

$$
\frac{\partial \Psi_{0}}{\partial x}=-\rho_{0} V_{0}, \quad \frac{\partial \Psi_{0}}{\partial Y}=\rho_{0} U_{0}
$$

Asymptotic analysis of the boundary-layer equations in region $B$ is based on the limit

$$
\zeta=\frac{Y}{s^{1 / 3}}=O(1), \quad s=x-1 \rightarrow 0^{+} .
$$

Asymptotic expansions for the stream function, enthalpy, density and viscosity are written in this region as

$$
\left.\begin{array}{rl}
\Psi_{0}(x, Y)=s^{2 / 3} g(\zeta)+\cdots, & h_{0}(x, Y)=h_{w}+s^{2 / 3} \bar{h}(\zeta)+\cdots, \\
\rho_{0}(x, Y)=\rho_{w}+s^{2 / 3} \bar{\rho}(\zeta)+\cdots, & \mu_{0}(x, Y)=\mu_{w}+s^{2 / 3} \bar{\mu}(\zeta)+\cdots,
\end{array}\right\}
$$

and it follows from the first of equations (5.5) that

$$
U_{0}=s^{1 / 3} \frac{1}{\rho_{w}} g^{\prime}(\zeta)+\cdots
$$

Substitution of (5.6) into the momentum equation $(2.2 a)$ yields

$$
\frac{1}{3} g^{2}-\frac{2}{3} g g^{\prime \prime}=\mu_{w} g^{\prime \prime \prime} .
$$

This equation should be solved with the symmetry conditions on the wake axis

$$
g(0)=g^{\prime \prime}(0)=0,
$$

and the condition of matching with the solution in region $A$

$$
g^{\prime}(\zeta)=\lambda \rho_{w} \zeta+\cdots \quad \text { as } \zeta \rightarrow \infty,
$$

which is deduced by comparing (5.7) with formulae (5.3), (5.4) representing $U_{0}$ in region $A$.

Solving the boundary-value problem (5.8)-(5.10) and using formula (5.7) gives the sought initial profile of the velocity in front of the interaction region. 


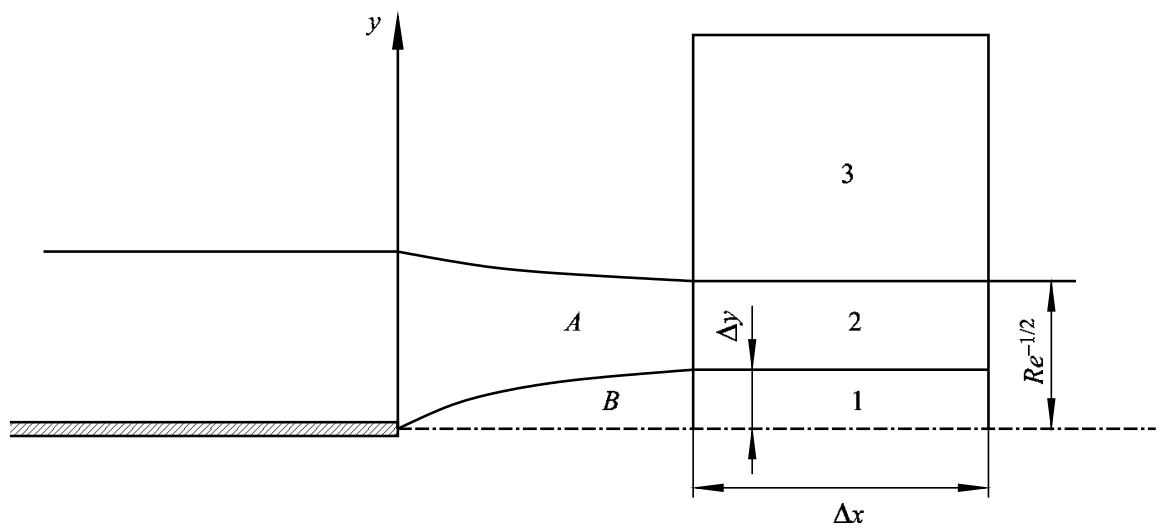

FIGURE 10. Three-tiered structure of the interaction region.

\subsection{Shock/wake interaction region}

The interaction region that forms in a vicinity of point $S$ (see figure 1) where the shock wave impinges upon the wake has the three-tiered structure shown in figure 10. Region 1 represents a continuation of region $B$ which means that its thickness may be estimated as $\Delta y \sim R e^{-1 / 2} Y \sim R e^{-1 / 2} s^{1 / 3}$. Taking into account that at the shock position $s=l_{s}$ we have

$$
\Delta y \sim R e^{-1 / 2} l_{s}^{1 / 3} .
$$

Region 2, being a continuation of region $A$, occupies the main body of the boundary layer, $y \sim R e^{-1 / 2}$. These should be supplemented by region 3 that lies outside the wake and serves to 'convert' the perturbations in the slope of the streamlines at the outer edge of region 2 into perturbations of pressure.

We first perform an order-of-magnitude estimation of fluid functions in the interaction region, which is termed after Sychev et al. (1998) an 'inspection' analysis. Starting with region 1 we can note that according to (5.7) the longitudinal velocity component may be estimated in this region as

$$
u \sim l_{s}^{1 / 3} .
$$

Since our goal is to investigate the process of the wake breakdown, we have to assume that the velocity perturbations $\Delta u \sim u$, i.e. the flow in region 1 is fully nonlinear. Therefore the shock strength should be such that

$$
\Delta p \sim u^{2} \sim l_{s}^{2 / 3} .
$$

Using the continuity equation we have

$$
\frac{\Delta u}{\Delta x} \sim \frac{v}{\Delta y} \Longrightarrow v \sim \frac{R e^{-1 / 2} l_{s}^{2 / 3}}{\Delta x},
$$

where $\Delta x$ denotes the longitudinal extent of the interaction region. The slope of the streamlines generated by the flow deceleration in region 1 may be estimated as

$$
\vartheta \sim \frac{v}{u} \sim \frac{R e^{-1 / 2} l_{s}^{1 / 3}}{\Delta x} .
$$

Using the order-of-magnitude arguments for the flow in the middle tier (region 2) it may be shown that the streamline slope does not change across region 2 . Therefore this formula may be also used to estimate the streamline slope at the 'bottom' of 
region 3. In this region the induced pressure can be calculated using the Ackeret formula, which gives

$$
\Delta p \sim \vartheta \sim \frac{R e^{-1 / 2} l_{s}^{1 / 3}}{\Delta x} .
$$

Comparing (5.11) with (5.12) we find that the longitudinal extent of the interaction region

$$
\Delta x \sim R e^{-1 / 2} l_{s}^{-1 / 3} .
$$

For all $l_{s} \gg R e^{-3 / 8}$ the separation $l_{s}$ of the interaction region from the trailing edge appears to be much larger than its size $\Delta x$.

Turning to rigorous asymptotic analysis of the flow in the interaction region we introduce a small parameter

$$
\varepsilon=l_{s}^{1 / 3}
$$

and represent the solution in the lower tier (region 1) in the form of the asymptotic expansions

$$
\left.\begin{array}{rl}
u(x, y, \operatorname{Re} ; \varepsilon)=\varepsilon U^{*}\left(X_{*}, Y_{*}\right)+\cdots, & v(x, y, \operatorname{Re} ; \varepsilon)=\varepsilon V^{*}\left(X_{*}, Y_{*}\right)+\cdots, \\
p(x, y ; \operatorname{Re}, \varepsilon)=\varepsilon^{2} P^{*}\left(X_{*}, Y_{*}\right)+\cdots, & \rho(x, y ; \operatorname{Re}, \varepsilon)=\rho_{w}+O\left(\varepsilon^{2}\right), \\
h(x, y ; \operatorname{Re}, \varepsilon)=h_{w}+O\left(\varepsilon^{2}\right), & \mu(x, y ; \operatorname{Re}, \varepsilon)=\mu_{w}+O\left(\varepsilon^{2}\right),
\end{array}\right\}
$$

where the scaled independent variables $X_{*}, Y_{*}$ are introduced by means of

$$
x=1+\varepsilon^{3}+R e^{-1 / 2} \varepsilon^{-1} X_{*}, \quad y=R e^{-1 / 2} \varepsilon Y_{*} .
$$

Substitution of (5.13), (5.14) into the Navier-Stokes equations shows that $P^{*}$ does not depend on $Y_{*}$ and reduces the momentum and continuity equations to

$$
\begin{gathered}
\rho_{w} U^{*} \frac{\partial U^{*}}{\partial X_{*}}+\rho_{w} V^{*} \frac{\partial U^{*}}{\partial Y_{*}}=-\frac{\partial P^{*}}{\partial X_{*}}, \\
\frac{\partial U^{*}}{\partial X_{*}}+\frac{\partial V^{*}}{\partial Y_{*}}=0 .
\end{gathered}
$$

Notice that the flow in region 1 appears to be inviscid.

The initial condition for equations (5.15)

$$
U^{*}=\frac{1}{\rho_{w}} g^{\prime}\left(Y_{*}\right)+\cdots \quad \text { as } \quad X_{*} \rightarrow-\infty
$$

follows from matching with solution (5.7) in region $B$.

To complete the formulation of the problem we need to supplement equations (5.15), (5.16) with the interaction law. It is deduced by analysing the flow in region 3 and its counterpart, region $3^{\prime}$, situated below the wake. Similar to (2.22), (2.25) we can write

$$
\begin{aligned}
& P_{+}^{*}=2 \bar{P}_{s} H\left(X_{*}-\bar{X}_{s}\right)+\frac{1}{\sqrt{M_{\infty}^{2}-1}} \lim _{Y_{s} \rightarrow \infty} \frac{V^{*}}{U^{*}}, \\
& P_{-}^{*}=-\frac{1}{\sqrt{M_{\infty}^{2}-1}} \lim _{Y_{s} \rightarrow-\infty} \frac{V^{*}}{U^{*}},
\end{aligned}
$$

and since the pressure does not change across the wake,

$$
P_{+}^{*}=P_{-}^{*} \quad \text { for all } \quad X_{*} \in(-\infty, \infty) .
$$


The interaction problem (5.15)-(5.17) can be converted, as before, into symmetrical form. For this purpose the Prandtl transposition is used

$$
\begin{gathered}
\bar{X}=X_{*}, \quad \bar{Y}=Y_{*}-F(\bar{X}), \\
U^{*}=\bar{U}, \quad V^{*}=\bar{V}+\frac{\mathrm{d} F}{\mathrm{~d} \bar{X}} \bar{U}, \quad P^{*}=\bar{P},
\end{gathered}
$$

where the function $F(\bar{X})$ should be chosen to be

$$
F(\bar{X})= \begin{cases}0 & \text { if } \bar{X}<\bar{X}_{s}, \\ -\sqrt{M_{\infty}^{2}-1} \bar{P}_{s}\left(\bar{X}-\bar{X}_{s}\right) & \text { if } \bar{X} \geqslant \bar{X}_{s},\end{cases}
$$

The momentum $(5.15 a)$ and continuity $(5.15 b)$ equations are invariant with respect to this transformation

$$
\begin{gathered}
\bar{U} \frac{\partial \bar{U}}{\partial \bar{X}}+\bar{V} \frac{\partial \bar{U}}{\partial \bar{Y}}=-\frac{1}{\rho_{w}} \frac{\mathrm{d} \bar{P}}{\mathrm{~d} \bar{X}}, \\
\frac{\partial \bar{U}}{\partial \bar{X}}+\frac{\partial \bar{V}}{\partial \bar{Y}}=0 .
\end{gathered}
$$

The initial condition (5.16) also remains unchanged

$$
\bar{U}=\frac{1}{\rho_{w}} g^{\prime}(\bar{Y})+\cdots \quad \text { as } \quad \bar{X} \rightarrow-\infty .
$$

The symmetry condition may be written as

$$
\bar{V}=0 \quad \text { at } \quad \bar{Y}=0,
$$

and, finally, the interaction law (5.17), (5.18) turns into

$$
\bar{P}=\bar{P}_{s} H\left(\bar{X}-\bar{X}_{s}\right)+\frac{1}{\sqrt{M_{\infty}^{2}-1}} \lim _{\bar{Y} \rightarrow \infty} \frac{\bar{V}}{\bar{U}} .
$$

In order to solve the interaction problem (5.19) we shall use the Von Mises' variables. Based on the continuity equation $(5.19 b)$ we introduce the stream function $\bar{\psi}$ such that

$$
\frac{\partial \bar{\psi}}{\partial \bar{Y}}=\rho_{w} \bar{U}, \quad \frac{\partial \bar{\psi}}{\partial \bar{X}}=-\rho_{w} \bar{V},
$$

and instead of $(\bar{X}, \bar{Y})$ use as independent variables $(\bar{X}, \bar{\psi})$. This transformation converts the momentum equation $(5.19 a)$ to

$$
\bar{U} \frac{\partial \bar{U}}{\partial \bar{X}}=-\frac{1}{\rho_{w}} \frac{\mathrm{d} \bar{P}}{\mathrm{~d} \bar{X}} .
$$

Being integrated, it yields the Bernoulli equation

$$
\frac{1}{2} \bar{U}^{2}+\frac{\bar{P}}{\rho_{w}}=\frac{1}{2}\left[\bar{U}_{0}(\bar{\psi})\right]^{2},
$$

where function $\bar{U}_{0}(\bar{\psi})$ represents the initial velocity profile $(5.19 c)$ expressed via the stream function $\bar{\psi}$.

The continuity equation (5.19b), written in the Von Mises' variables, has the form

$$
\frac{\partial}{\partial \bar{\psi}}\left(\frac{\bar{V}}{\bar{U}}\right)=\frac{1}{\rho_{w}} \frac{\partial}{\partial \bar{X}}\left(\frac{1}{\bar{U}}\right) .
$$


Solving the Bernoulli equation (5.21) for $\bar{U}$

$$
\bar{U}=\sqrt{\left[\bar{U}_{0}(\bar{\psi})\right]^{2}-\frac{2}{\rho_{w}} \bar{P}(\bar{X})}
$$

and using (5.23) to calculate the right hand side in (5.22) we can find, after integrating with respect to $\bar{\psi}$, that the streamline slope angle

$$
\frac{\bar{V}}{\bar{U}}=\frac{1}{\rho_{w}^{2}} \frac{\mathrm{d} \bar{P}}{\mathrm{~d} \bar{X}} \int_{0}^{\bar{\psi}}\left\{\left[\bar{U}_{0}(\psi)\right]^{2}-\frac{2}{\rho_{w}} \bar{P}(\bar{X})\right\}^{-3 / 2} \mathrm{~d} \psi .
$$

It remains to set $\bar{\psi}=\infty$ in (5.24) and substitute the resulting formula into the interaction law $(5.19 e)$. We arrive at the following integro-differential equation:

$$
\bar{P}=\bar{P}_{s} H\left(\bar{X}-\bar{X}_{s}\right)+\frac{1}{\rho_{w}^{2} \sqrt{M_{\infty}^{2}-1}} \frac{\mathrm{d} \bar{P}}{\mathrm{~d} \bar{X}} \int_{0}^{\infty}\left\{\left[\bar{U}_{0}(\bar{\psi})\right]^{2}-\frac{2}{\rho_{w}} \bar{P}(\bar{X})\right\}^{-3 / 2} \mathrm{~d} \bar{\psi}
$$

for the pressure $\bar{P}(\bar{X})$.

When solving this equation one needs to know the initial velocity profile $\bar{U}_{0}(\bar{\psi})$ in terms of the stream function $\bar{\psi}$. In the original variables it is given by $(5.19 c)$. Comparing (5.19c) with the first of equations (5.20) we can find that $\bar{\psi}=g(\bar{Y})$. Hence, the initial velocity profile $\bar{U}_{0}(\bar{\psi})$ may be written in the following implicit form

$$
\bar{U}_{0}(\bar{\psi})=\frac{1}{\rho_{w}} g^{\prime}(\bar{Y}), \quad \bar{\psi}=g(\bar{Y}) .
$$

Guided by (5.26) we return to equation (5.8) and seek its solution in the form

$$
g^{\prime}(\bar{Y})=\rho_{w} \bar{U}[g(\bar{Y})] .
$$

Substitution of (5.27) into (5.8) results in the following equation for $\bar{U}(\bar{\psi})$ :

$$
\frac{1}{3} \bar{U}_{0}^{2}-\frac{1}{3} \bar{\psi}\left(\bar{U}_{0}^{2}\right)^{\prime}=\frac{1}{2} \mu_{w} \rho_{w} \bar{U}_{0}\left(\bar{U}_{0}^{2}\right)^{\prime \prime},
$$

with the boundary conditions (5.9), (5.10) turning to

$$
\begin{aligned}
& \bar{U}_{0}^{\prime}=0 \text { at } \bar{\psi}=0, \\
& \bar{U}_{0}=\sqrt{\frac{2 \lambda}{\rho_{w}} \bar{\psi}}+\cdots \quad \text { as } \bar{\psi} \rightarrow \infty .
\end{aligned}
$$

The affine transformations

$$
\bar{U}_{0}=\lambda^{2 / 3} \rho_{w}^{-1 / 3} \mu_{w}^{1 / 3} u_{0}, \quad \bar{\psi}=\lambda^{1 / 3} \rho_{w}^{1 / 3} \mu_{w}^{2 / 3} \psi
$$

allow parameters $\mu_{w}, \rho_{w}$ and $\lambda$ to be excluded from the formulation of the boundaryvalue problem (5.28), (5.29). We have

$$
\begin{aligned}
\frac{2}{3} u_{0}^{2}-\frac{2}{3} \psi\left(u_{0}^{2}\right)^{\prime}=u_{0}\left(u_{0}^{2}\right)^{\prime \prime}, \\
u_{0}^{\prime}=0 \quad \text { at } \quad \psi=0, \\
u_{0}=\sqrt{2 \psi}+\cdots \quad \text { as } \quad \psi \rightarrow \infty .
\end{aligned}
$$

If in addition to (5.30) we use transformations

$$
\bar{P}=\lambda^{4 / 3} \rho_{w}^{1 / 3} \mu_{w}^{2 / 3} p, \quad \bar{X}=\bar{X}_{s}+\lambda^{-5 / 3} \rho_{w}^{-2 / 3} \mu_{w}^{-1 / 3}\left(M_{\infty}^{2}-1\right)^{-1 / 2} x,
$$


then the integro-differential equation (5.25) takes the following canonical form:

$$
p=p_{s} H(x)+\frac{\mathrm{d} p}{\mathrm{~d} x} \int_{0}^{\infty}\left(u_{0}^{2}-2 p\right)^{-2 / 3} \mathrm{~d} \psi,
$$

where

$$
p_{s}=\frac{\bar{P}_{s}}{\lambda^{4 / 3} \rho_{w}^{1 / 3} \mu_{w}^{2 / 3}}
$$

proves to be the only non-dimensional parameter that governs the flow in the interaction region.

Similar to the triple-deck problem (2.34) we shall impose on the solution of equation (5.32a) the following downstream boundary condition

$$
p=p_{s} \quad \text { at } \quad x=\infty .
$$

When solving (5.32a) one has to keep in mind that according to the Bernoulli equation (5.21) the pressure rise in the interaction region is restricted by the condition

$$
\left[\bar{U}_{0}(\bar{\psi})\right]^{2}-\frac{2}{\rho_{w}} \bar{P}(\bar{X}) \geqslant 0 .
$$

Taking into account that the initial velocity profile has its minimum at the wake axis, we can conclude that the critical value of the shock strength

$$
\bar{P}_{s}^{*}=\frac{\rho_{w}}{2}\left[\bar{U}_{0}(0)\right]^{2}
$$

Using further affine transformations (5.30), (5.31) yields the critical value of parameter $p_{s}$ :

$$
p_{s}^{*}=\left.\frac{1}{2} u_{0}^{2}\right|_{\psi=0}
$$

beyond which the solution of equation (5.32a), apparently, does not exist.

The results of the numerical solution of problem (5.32) are displayed in figure 11 . We found that for all $p_{s}<p_{s}^{*}$ the pressure grows monotonically upstream of the shock. At the shock position the derivative $\mathrm{d} p / \mathrm{d} x$ has a discontinuity, and the pressure remains constant and equal to $p_{s}$ everywhere downstream of the shock. When the parameter $p_{s}$ approaches the critical value $p_{s}^{*}$, the perturbations start to propagate very fast in the upstream direction. It is interesting to notice that the solutions corresponding to $p_{s} / p_{s}^{*}=0.99 ; 0.999$ and 0.9999 are essentially the same. The only difference between them is a shift in the $x$-direction. By this stage the distribution of pressure (and, hence, all other functions) takes a universal form characteristic of the so-called 'self-induced wake breakdown' which will be considered in the next section.

Before doing this we shall return to the triple-deck calculations of $\S 4$, and observe that the wake breakdown prediction based on the Bernoulli equation may be directly linked to the hysteresis behaviour of the trailing-edge flow, as shown in figures 5 and 6. If, with fixed shock position $X_{s}>3.2$, we monotonically increase the shock strength $P_{s}$, then as long as the solution remains on the upper branch the flow in the wake experiences a deceleration but the velocity $U$ remains positive everywhere. This type of solution becomes impossible when the shock strength $P_{s}$ reaches a critical value, which we shall denote as $P_{s}^{\star}$, and the solution 'jumps' to the lower branch with an extended region of flow reversal. It is shown for different $X_{s}$ in figures $8(e)$ and $8(f)$; the breakdown point being situated some distance upstream of $X_{s}$. 


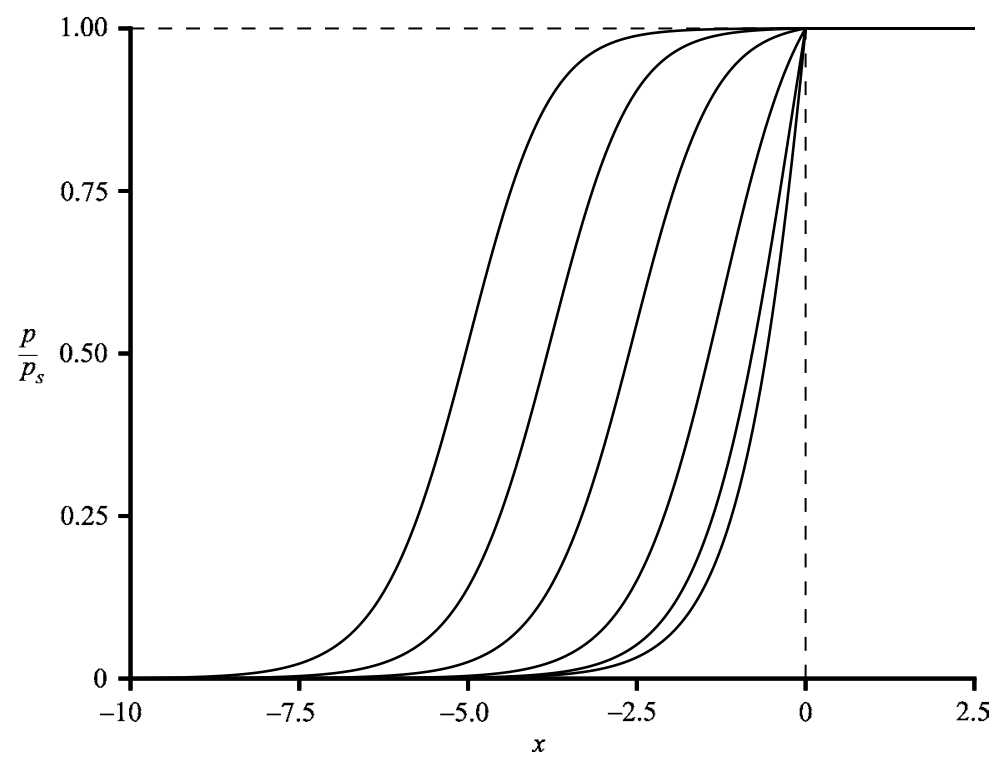

FIGURE 11. Results of numerical solution of equation $(5.32 a)$ with boundary condition $(5.32 b)$. The six curves shown correspond to, from right to left, $p_{s} / p_{s}^{*}=0.3 ; 0.6 ; 0.9 ; 0.99 ; 0.999$ and 0.9999 .

$\begin{array}{ccc}x_{s} & P_{s}^{*} & P_{s}^{\star} \\ 3.4 & 2.86052 & 2.71774 \\ 3.8 & 3.08616 & 2.95223 \\ 4.2 & 3.30416 & 3.19986 \\ 4.6 & 3.51546 & 3.44665\end{array}$

TABLE 2. Hysteresis predictions from Bernoulli equation.

In table 2 we compare $P_{s}^{\star}$ with the critical shock strength $P_{s}^{*}$ calculated based on the Bernoulli equation

$$
P_{s}^{*}=\frac{1}{2}\left[U\left(X_{s}, 0\right)\right]^{2} \text {. }
$$

Here $U\left(X_{s}, 0\right)$ is the velocity on the wake axis at the shock position $X_{s}$ calculated using the triple-deck code of $\S 3$ in the flow with no impinging shock $\left(P_{s}=0\right)$.

It can be seen that the value found from our triple-deck calculations, $P_{s}^{\star}$, is close to that predicted by Bernoulli's equation which bounds it from above. As we move the shock further downstream $P_{s}^{\star}$ and $P_{s}^{\star}$ appear to converge confirming the shock/wake interaction becomes predominantly inviscid. This suggests, interestingly enough, that the Bernoulli equation may be used as a simple practical tool for predicting the wake breakdown.

It is also interesting to notice that the pressure inside the wake appears to be continuous both in the inviscid-inviscid interaction flow regime considered here and in the case of classical viscous-inviscid interaction discussed in $\S \S 2-4$; see the pressure curve in figure 4 . Of course, due to the presence of the shock the flow field remains discontinuous everywhere above the wake. However, as the wake is approached the shock becomes progressively weaker, being compensated by the reaction of the flow inside the wake. The fact is that, as long the velocity on the wake axis is small as 
compared to the free-stream velocity, the wake may be shown to be incapable of sustaining an infinite pressure gradient that would be observed if the shock could penetrate into the wake. It therefore adjusts itself in such a way that the discontinuity brought about by the first term on the right hand side of (3.10) is annulled by the second term representing the displacement effect of the boundary layer.

\section{Self-induced wake breakdown}

Here we shall assume that the shock strength exceeds the critical value, and an extended region of flow reversal forms in the wake with the breakdown point situated some distance upstream of the shock position. If this distance is much larger than the extent of the inviscid-inviscid interaction region, that forms around the breakdown point, then the process of fluid deceleration in the wake may be described by equation (5.25) without the shock term:

$$
\bar{P}=\frac{1}{\rho_{w}^{2} \sqrt{M_{\infty}^{2}-1}} \frac{\mathrm{d} \bar{P}}{\mathrm{~d} \bar{X}} \int_{0}^{\infty}\left\{\left[\bar{U}_{0}(\bar{\psi})\right]^{2}-\frac{2}{\rho_{w}} \bar{P}(\bar{X})\right\}^{-3 / 2} \mathrm{~d} \bar{\psi} .
$$

We shall first concentrate on finding the asymptotic behaviour of the solution of (6.1) as the flow approaches the breakdown point.

\subsection{Asymptotic solution of inviscid-inviscid interaction equation}

Using arguments similar to those in Watson's lemma, it may be proven that the dominant contribution to the integral

$$
I=\int_{0}^{\infty}\left\{\left[\bar{U}_{0}(\bar{\psi})\right]^{2}-\frac{2}{\rho_{w}} \bar{P}(\bar{X})\right\}^{-3 / 2} \mathrm{~d} \bar{\psi}
$$

is given by a small vicinity of the wake axis, where

$$
\bar{U}_{0}(\bar{\psi})=a+b \bar{\psi}^{2}+\cdots \quad \text { as } \quad \bar{\psi} \rightarrow 0 ;
$$

both constants $a$ and $b$ being positive.

To prove this statement, we write the integral in the form

$$
I=\int_{0}^{\infty}\left\{\left[\bar{U}_{0}(\bar{\psi})\right]^{2}-a^{2}+\left[a^{2}-\frac{2}{\rho_{w}} \bar{P}(\bar{X})\right]\right\}^{-3 / 2} \mathrm{~d} \bar{\psi}
$$

and assume that

$$
\epsilon(\bar{X})=\sqrt{a^{2}-\frac{2}{\rho_{w}} \bar{P}(\bar{X})}
$$

tends to zero. We then introduce $\delta(\bar{X})$ such that $\epsilon \ll \delta \ll 1$, and split the integral (6.4) into two:

$$
I=\int_{0}^{\delta}\left\{\left[\bar{U}_{0}(\bar{\psi})\right]^{2}-a^{2}+\epsilon^{2}\right\}^{-3 / 2} \mathrm{~d} \bar{\psi}+\int_{\delta}^{\infty}\left\{\left[\bar{U}_{0}(\bar{\psi})\right]^{2}-a^{2}+\epsilon^{2}\right\}^{-3 / 2} \mathrm{~d} \bar{\psi} .
$$

Since $\delta \ll 1$, the first integral may be calculated using asymptotic expansion (6.3). Disregarding $O\left(\bar{\psi}^{4}\right)$ terms, we have

$$
I_{1}=\int_{0}^{\delta}\left\{\left[\bar{U}_{0}(\bar{\psi})\right]^{2}-a^{2}+\epsilon^{2}\right\}^{-3 / 2} \mathrm{~d} \bar{\psi}=\int_{0}^{\delta} \frac{\mathrm{d} \bar{\psi}}{\left(2 a b \bar{\psi}^{2}+\epsilon^{2}\right)^{-3 / 2}} .
$$


In this integral the ballance between $2 a b \bar{\psi}^{2}$ and $\epsilon^{2}$ is important, which is why (with $\delta$ being much larger than $\epsilon$ ) the integration interval $\bar{\psi} \in[0, \delta]$ covers the entire region of dominant contribution in (6.2). Keeping this in mind, we introduce a new integration variable $s=\bar{\psi} / \epsilon$, and calculate integral (6.6) as

$$
I_{1}=\frac{1}{\epsilon^{2}} \int_{0}^{\delta / \epsilon} \frac{\mathrm{d} s}{\left(2 a b s^{2}+1\right)^{3 / 2}}=\left.\frac{1}{\epsilon^{2}} \frac{s}{\sqrt{2 a b s^{2}+1}}\right|_{0} ^{\delta / \epsilon}=\frac{1}{\epsilon^{2} \sqrt{2 a b}} .
$$

Turning to the second integral

$$
I_{2}=\int_{\delta}^{\infty}\left\{\left[\bar{U}_{0}(\bar{\psi})\right]^{2}-a^{2}+\epsilon^{2}\right\}^{-3 / 2} \mathrm{~d} \bar{\psi}
$$

we note that both $\left[\bar{U}_{0}(\bar{\psi})\right]^{2}-a^{2}$ and $\epsilon^{2}$ are positive. Consequently, $I_{2}$ may be bounded as

$$
\left|I_{2}\right| \leqslant \int_{\delta}^{\infty} \frac{\mathrm{d} \bar{\psi}}{\left\{\left[\bar{U}_{0}(\bar{\psi})\right]^{2}-a^{2}\right\}^{3 / 2}} .
$$

We then divide the integral in (6.8) into two:

$$
\int_{\delta}^{\infty} \frac{\mathrm{d} \bar{\psi}}{\left\{\left[\bar{U}_{0}(\bar{\psi})\right]^{2}-a^{2}\right\}^{3 / 2}}=\left\{\int_{\delta}^{1}+\int_{1}^{\infty}\right\} \frac{\mathrm{d} \bar{\psi}}{\left\{\left[\bar{U}_{0}(\bar{\psi})\right]^{2}-a^{2}\right\}^{3 / 2}} .
$$

Taking into account that $\bar{U}_{0}(\bar{\psi})$ grows monotonically with $\bar{\psi}$, and at small $\bar{\psi}$ is given by (6.3), one can see that there exists constant $A>0$ such that

$$
\left[\bar{U}_{0}(\bar{\psi})\right]^{2}-a^{2} \geqslant A \bar{\psi}^{2} \quad \text { for } \quad \bar{\psi} \in[0,1] .
$$

Hence,

$$
\left|\int_{\delta}^{1} \frac{\mathrm{d} \bar{\psi}}{\left\{\left[\bar{U}_{0}(\bar{\psi})\right]^{2}-a^{2}\right\}^{3 / 2}}\right| \leqslant \frac{1}{A^{3 / 2}} \int_{\delta}^{\infty} \frac{\mathrm{d} \bar{\psi}}{\bar{\psi}^{3}}=\frac{1}{2 A^{3 / 2}}\left(\frac{1}{\delta^{2}}-1\right) .
$$

The second integral in (6.9) is finite, and therefore, for any $\delta \ll 1$ appears to be much smaller than the first integral (6.10). The latter is obviously small compared to (6.7) as long as $\epsilon \ll \delta$.

Therefore, substituting (6.5) into (6.7) we can finally write

$$
\int_{0}^{\infty}\left\{\left[\bar{U}_{0}(\bar{\psi})\right]^{2}-\frac{2}{\rho_{w}} \bar{P}(\bar{X})\right\}^{-3 / 2} \mathrm{~d} \bar{\psi}=\frac{1}{\sqrt{2 a b}\left[a^{2}-\left(2 / \rho_{w}\right) \bar{P}(\bar{X})\right]} .
$$

With (6.11) equation (6.1) takes the form

$$
\bar{P}=\frac{1}{\rho_{w}^{2} \beta \sqrt{2 a b}\left[a^{2}-\left(2 / \rho_{w}\right) \bar{P}(\bar{X})\right]} \frac{\mathrm{d} \bar{P}}{\mathrm{~d} \bar{X}},
$$

where $\beta=\sqrt{M_{\infty}^{2}-1}$. This equation is easily integrated using the separation of variables. Its solution has the form

$$
\bar{P}=\frac{1}{2} \rho_{w} a^{2} \frac{1}{1+\mathrm{e}^{-\kappa \bar{X}+C}},
$$

where $\kappa=\sqrt{2} \rho_{w}^{2} \beta a^{5 / 2} b^{1 / 2}$. Hence, the sought asymptotic behaviour of pressure is given by

$$
\bar{P}=\frac{1}{2} \rho_{w} a^{2}\left(1-\mathrm{e}^{-\kappa \bar{X}}\right)+\cdots \quad \text { as } \quad \bar{X} \rightarrow \infty .
$$


Here it has been taken into account that (6.1) is invariant with respect to an arbitrary shift in $\bar{X}$-direction, and therefore, without loss of generality the constant of integration $C$ may be disregarded in (6.12).

With known pressure all other functions in the inviscid-inviscid interaction region may be easily found. In particular, substitution of (6.3) and (6.13) into (5.23) yields

$$
\bar{U}=\exp \left(-\frac{1}{2} \kappa \bar{X}\right) \sqrt{a^{2}+2 a b \xi^{2}}+\cdots \quad \text { as } \quad \bar{X} \rightarrow \infty .
$$

Here

$$
\xi=\frac{\bar{\psi}}{\exp \left(-\frac{1}{2} \kappa \bar{X}\right)}
$$

has to be assumed an order-one quantity if the entire region of dominant contribution to the displacement effect of the wake is to be considered.

\subsection{Viscous region}

Formula (5.23) shows that inviscid theory is incapable of describing the flow reversal characteristic of the wake breakdown. At the same time it follows from (6.12) that the fluid motion becomes very slow as $\bar{X}$ increases, and therefore viscosity effects have to be taken into account near the point of breakdown. We shall concentrate our attention on the region of slow motion that gives the main contribution to the displacement effect of the wake. In this region $\xi=O(1)$, and it follows from (6.14) and (6.15) that

$$
\bar{U}=O\left[\exp \left(-\frac{1}{2} \kappa \bar{X}\right)\right], \quad \bar{\psi}=O\left[\exp \left(-\frac{1}{2} \kappa \bar{X}\right)\right] .
$$

Hence, using the first of equations (5.20), we can conclude that

$$
\bar{Y}=O(1) .
$$

Let us now compare the pressure gradient with the "main" viscous term in the $x$-momentum Navier-Stokes equation

$$
\frac{\partial p}{\partial x} \sim \frac{1}{R e} \frac{\partial^{2} u}{\partial y^{2}} .
$$

Using (5.13), (5.14) and (6.13) we can find that

$$
\frac{\partial p}{\partial x}=\varepsilon^{3} R e^{1 / 2} \frac{\partial P^{*}}{\partial X^{*}}=\varepsilon^{3} R e^{1 / 2} \frac{\partial \bar{P}}{\partial \bar{X}} \sim \varepsilon^{3} R e^{1 / 2} \mathrm{e}^{-\kappa \bar{X}} .
$$

Similarly, it follows from (5.13), (5.14), (6.14) and (6.16) that

$$
\frac{1}{R e} \frac{\partial^{2} u}{\partial y^{2}}=\frac{1}{\varepsilon} \frac{\partial^{2} U^{*}}{\partial Y^{* 2}}=\frac{1}{\varepsilon} \frac{\partial^{2} \bar{U}}{\partial \bar{Y}} \sim \frac{1}{\varepsilon} \exp \left(-\frac{1}{2} \kappa \bar{X}\right) .
$$

Substituting (6.18) and (6.19) into (6.17), we see that the balance between the pressure gradient and viscous term holds if

$$
e^{\frac{1}{2} \kappa \bar{X}}=\Lambda \varepsilon^{4} R e^{1 / 2},
$$

where $\Lambda$ is an order one quantity.

Solving the above equation for $\bar{X}$ we find

$$
\bar{X}=\frac{2}{\kappa} \ln \left(\varepsilon^{4} R e^{1 / 2}\right)+\frac{2}{\kappa} \ln \Lambda,
$$


which suggests that the characteristic longitudinal variable $X^{\circ}$ in the viscous region should be defined by the equation

$$
\bar{X}=\frac{2}{\kappa} \ln \left(\varepsilon^{4} R e^{1 / 2}\right)+X^{\circ} .
$$

Substituting (6.20) into (6.15) and taking into account that $\xi=O(1)$ we can see that the scaled stream function in the viscous region $\psi^{\circ}$ has to be such that

$$
\bar{\psi}=\varepsilon^{-4} R e^{-1 / 2} \psi^{\circ} \text {. }
$$

Substitution of (6.20) and (6.21) into (6.14) and then into the asymptotic expansion for $u(x, y ; R e, \varepsilon)$ in $(5.13)$ yields

$$
u(x, y ; R e, \varepsilon)=\varepsilon^{-3} R e^{-1 / 2} \exp \left(-\frac{1}{2} \kappa X^{\circ}\right) \sqrt{a^{2}+2 a b \xi^{2}}+\cdots,
$$

which means that in the viscous region the longitudinal velocity component should be sought in the form of asymptotic expansion

$$
u(x, y ; R e, \varepsilon)=\varepsilon^{-3} \operatorname{Re}^{-1 / 2} U^{\circ}+\cdots,
$$

where function $U^{\circ}$ satisfies the following matching condition

$$
U^{\circ}=\exp \left(-\frac{1}{2} \kappa X^{\circ}\right) \sqrt{a^{2}+2 a b \xi^{2}}+\cdots \quad \text { as } \quad X^{\circ} \rightarrow-\infty .
$$

The self-similar variable (6.15) may be calculated using (6.20), (6.21) as

$$
\xi=\frac{\psi^{\circ}}{\exp \left(-\frac{1}{2} \kappa X^{\circ}\right)} .
$$

Using similar arguments for pressure we can find from (6.13) that in the viscous region

$$
p(x, y ; R e, \varepsilon)=\varepsilon^{2} \frac{1}{2} \rho_{w} a^{2}+\varepsilon^{-6} R e^{-1} P^{\circ}+\cdots,
$$

with the matching condition for $P^{\circ}$ being

$$
P^{\circ}=-\frac{1}{2} \rho_{w} a^{2} \exp \left(-\kappa X^{\circ}\right)+\cdots \quad \text { as } \quad X^{\circ} \rightarrow-\infty .
$$

Combining (5.14) with (6.16) and (6.20) we see that independent variables in the viscous region

$$
x=1+\varepsilon^{3}+\varepsilon^{-1} R e^{-1 / 2} \frac{2}{\kappa} \ln \left(\varepsilon^{4} R e^{1 / 2}\right)+\varepsilon^{-1} R e^{-1 / 2} X^{\circ}, \quad y=\varepsilon R e^{-1 / 2} Y^{\circ} .
$$

It remains to estimate the lateral velocity component. This may be done based on the continuity equation

$$
\frac{\partial v}{\partial y} \sim \frac{\partial u}{\partial x} \Longrightarrow v \sim u \frac{y}{\Delta x} \sim \varepsilon^{-1} R e^{-1 / 2}
$$

Summarising these results we seek the solution of the Navier-Stokes equations in the viscous region in the form

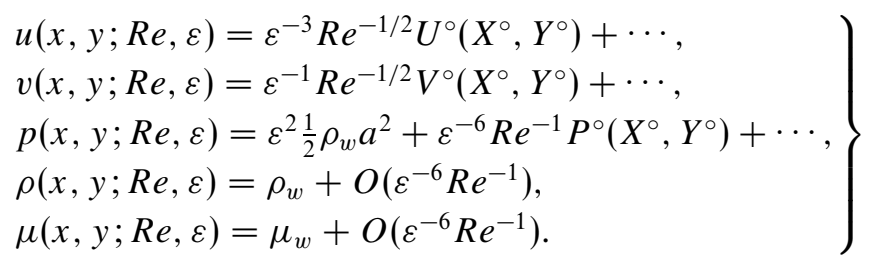


Substitution of (6.25) together with (6.24) into the Navier-Stokes equation results in the incompressible boundary-layer equations

$$
\left.\begin{array}{rl}
\rho_{w}\left(U^{\circ} \frac{\partial U^{\circ}}{\partial X^{\circ}}+V^{\circ} \frac{\partial U^{\circ}}{\partial Y^{\circ}}\right) & =-\frac{\mathrm{d} P^{\circ}}{\mathrm{d} X^{\circ}}+\mu_{w} \frac{\partial^{2} U^{\circ}}{\partial Y^{\circ 2}}, \\
\frac{\partial U^{\circ}}{\partial X^{\circ}}+\frac{\partial V^{\circ}}{\partial Y^{\circ}} & =0 .
\end{array}\right\}
$$

The initial condition for the longitudinal velocity component $U^{\circ}$ is given by $(6.22)$. To express it in Cartesian coordinates we substitute (6.23) into (6.22)

$$
U^{\circ}=\exp \left(-\frac{1}{2} \kappa X^{\circ}\right) \sqrt{a^{2}+2 a b \frac{\psi^{\circ 2}}{\exp \left(-\kappa X^{\circ}\right)}}+\cdots \quad \text { as } \quad X^{\circ} \rightarrow-\infty .
$$

and note that the stream function $\psi^{\circ}$ satisfies the equations

$$
\frac{\partial \psi^{\circ}}{\partial Y^{\circ}}=\rho_{w} U^{\circ}, \quad \frac{\partial \psi^{\circ}}{\partial X^{\circ}}=-\rho_{w} V^{\circ} .
$$

Combining the first of them with (6.27) yields

$$
\frac{\partial \psi^{\circ}}{\partial Y^{\circ}}=\rho_{w} \exp \left(-\frac{1}{2} \kappa X^{\circ}\right) \sqrt{a^{2}+2 a b \frac{\psi^{\circ 2}}{\exp \left(-\kappa X^{\circ}\right)}}+\cdots \quad \text { as } \quad X^{\circ} \rightarrow-\infty .
$$

This equation may be easily integrated for $\psi^{\circ}$ subject to the boundary condition $\psi^{\circ}=0$ at $Y^{\circ}-0$. We have

$$
\psi^{\circ}=\sqrt{\frac{a}{2 b}} \exp \left(-\frac{1}{2} \kappa X^{\circ}\right) \sinh \left(\rho_{w} \sqrt{2 a b} Y^{\circ}\right)+\cdots \quad \text { as } \quad X^{\circ} \rightarrow-\infty,
$$

and the sought initial condition for $U^{\circ}$ is written as

$$
U^{\circ}=a \exp \left(-\frac{1}{2} \kappa X^{\circ}\right) \cosh \left(\rho_{w} \sqrt{2 a b} Y^{\circ}\right)+\cdots \quad \text { as } \quad X^{\circ} \rightarrow-\infty,
$$

The boundary conditions on the wake axis are

$$
\frac{\partial U^{\circ}}{\partial Y^{\circ}}=V^{\circ}=0 \quad \text { at } \quad Y^{\circ}=0
$$

and to close the problem we have to formulate the interaction law. In the inviscid flow outside the wake the Ackeret formula

$$
p=\frac{\vartheta}{\beta}, \quad \text { where } \vartheta=\arctan \left(\frac{v}{u}\right)
$$

is valid. Substitution of (6.25) into (6.30) yields

$$
\lim _{Y^{\circ} \rightarrow \infty} \frac{V^{\circ}}{U^{\circ}}=\frac{1}{2} \beta \rho_{w} a^{2} \text {. }
$$

Thus the interaction problem consists in solving equations (6.26) with boundary conditions (6.28), (6.29) and interaction law (6.31). It resembles the corresponding problem formulated by Sychev, Vic. V. (1978) who studies the wake breakdown in incompressible fluid flow. It is remarkable that the solution may be written in analytical form

$$
U^{\circ}=a \exp \left(-\frac{1}{2} \kappa X^{\circ}\right) \cosh \left(\rho_{w} \sqrt{2 a b} Y^{\circ}\right)-\frac{4}{\kappa} \mu_{w} \rho_{w} a b,
$$




$$
\begin{aligned}
V^{\circ} & =\frac{\kappa a^{1 / 2}}{\rho_{w} \sqrt{8 b}} \exp \left(-\frac{1}{2} \kappa X^{\circ}\right) \sinh \left(\rho_{w} \sqrt{2 a b} Y^{\circ}\right), \\
P^{\circ} & =-\frac{\rho_{w} a^{2}}{2} \exp \left(-\kappa X^{\circ}\right) .
\end{aligned}
$$

It follows from the expression for $U^{\circ}$ that flow reversal first forms near the axis of symmetry, and then as $X^{\circ}$ increases, it spreads to a wider portion of the wake.

\section{Concluding remarks}

The analysis presented in this paper shows that the interaction of a shock wave with the wake downstream of a flat plate is a complex phenomenon. If the shock impinges upon the wake close to the trailing edge then the triple-deck theory may be used. In this case the flow is viscous and displays a hysteresis behaviour which, in our view, may be explained by a sharp acceleration on the background wake flow downstream of the trailing edge. The deceleration of fluid in the wake caused by the shock depends on its strength, but as long as the solution remains on the upper branch no flow reversal is observed in the wake core. However, when the shock strength reaches a critical value the solution has to jump to the lower branch. This is accompanied by complete reconstruction of the flow field. In particular, a reversed flow region suddenly forms, being of an 'adult size at the birth'.

If, on the other hand, the shock is situated further downstream, then the shock/wake interaction proves to be predominantly inviscid. Due to this simplification the interaction problem may be reduced to a single integro-differential equation governing the pressure distribution along the interaction region. We found that the pressure grows monotonically as the shock position is approached, and then remains constant everywhere downstream of the shock. Correspondingly, the fluid velocity inside the wake decays monotonically, as the Bernoulli equation predicts, and reaches its minimum on the wake axis at the shock position. This observation allows for a simple criterion of the wake breakdown to be formulated. The breakdown takes place when the shock strength is large enough to reduce the minimal velocity to zero. The accuracy of the predictions based on the Bernoulli equation may be improved, if necessary, by analysing a viscous region that forms in the wake near the point of flow reversal. The flow in this region is governed by the boundary-layer equations with prescribed displacement function. We found that the streamline slope and, hence, the pressure should remain constant in this region. It is remarkable that the solution of the corresponding boundary-value problem admits an analytical solution.

\section{Appendix}

In order to study asymptotic behaviour of the solution of equations (2.10) at large values of $Y_{*}$, it is convenient to introduce the stream function $\Psi^{*}\left(x_{*}, Y_{*}\right)$. Its existence follows from the continuity equation $(2.10 b)$, and we can write

$$
U^{*}=\frac{\partial \Psi^{*}}{\partial Y_{*}}, \quad V^{*}=-\frac{\partial \Psi^{*}}{\partial x_{*}} .
$$

Let us try to use for the stream function the following asymptotic expansion

$$
\Psi^{*}\left(x_{*}, Y_{*}\right)=A_{0}\left(x_{*}\right) Y_{*}^{\alpha}+\cdots \quad \text { as } \quad Y_{*} \rightarrow \infty .
$$


Here parameter $\alpha$ and function $A_{0}\left(x_{*}\right)$ are expected to be found by using the momentum equation $(2.10 a)$.

Substitution of (A 2) into (A 1) yields

$$
U^{*}=\alpha A_{0}\left(x_{*}\right) Y_{*}^{\alpha-1}+\cdots, \quad V^{*}=-A_{0}^{\prime}\left(x_{*}\right) Y_{*}^{\alpha}+\cdots .
$$

Therefore, the convective terms on the left hand side of $(2.10 a)$ and the viscous term on the right hand side are written as

$$
\begin{aligned}
\rho_{w} U^{*} \frac{\partial U^{*}}{\partial x_{*}} & =\rho_{w} \alpha A_{0} \frac{\mathrm{d} A_{0}}{\mathrm{~d} x_{*}} Y_{*}^{2 \alpha-2}+\cdots, \\
\rho_{w} V^{*} \frac{\partial U^{*}}{\partial Y_{*}} & =-\rho_{w} \alpha(\alpha-1) A_{0} \frac{\mathrm{d} A_{0}}{\mathrm{~d} x_{*}} Y_{*}^{2 \alpha-2}+\cdots, \\
\mu_{w} \frac{\partial^{2} U^{*}}{\partial Y_{*}^{2}} & =\alpha(\alpha-1)(\alpha-2) A_{0} Y_{*}^{\alpha-3}+\cdots
\end{aligned}
$$

We see that if we assume, subject to subsequent confirmation, that $\alpha>1$, then the convective terms will dominate not only over the viscous term, but also over the pressure gradient, which remains finite as $Y_{*} \rightarrow \infty$. We have

$$
O\left(Y_{*}^{2 \alpha-2}\right): \quad A_{0} \frac{\mathrm{d} A_{0}}{\mathrm{~d} x_{*}}=0 .
$$

The initial condition for this equation may be obtained by comparing the first of formulae (A 3) with (2.12). We have

$$
A_{0}(-\infty)=\left\{\begin{array}{lll}
\lambda / \alpha & \text { if } & \alpha=2 \\
0 & \text { if } & \alpha=2
\end{array}\right.
$$

Hence a non-trivial solution exist only if $\alpha=2$, in which case $A_{0}=\frac{1}{2} \lambda$, and (A 2) turns into

$$
\Psi^{*}\left(x_{*}, Y_{*}\right)=\frac{1}{2} \lambda Y_{*}^{2}+\cdots \quad \text { as } \quad Y_{*} \rightarrow \infty .
$$

Now we shall try to find the next order term in this expansion:

$$
\Psi^{*}\left(x_{*}, Y_{*}\right)=\frac{1}{2} \lambda Y_{*}^{2}+A_{1}\left(x_{*}\right) Y_{*}^{\alpha}+\cdots \quad \text { as } Y_{*} \rightarrow \infty .
$$

In order to ensure that the second term in (A 4) is small as compared with the first one, we have to assume that $\alpha<2$. Substitution of (A 4) into (A 1) yields

$$
U^{*}=\lambda Y_{*}+\alpha A_{1}\left(x_{*}\right) Y_{*}^{\alpha-1}+\cdots, \quad V^{*}=-A_{1}^{\prime}\left(x_{*}\right) Y_{*}^{\alpha}+\cdots .
$$

Therefore, the convective terms on the left hand side of $(2.10 a)$ and the viscous term on the right hand side are written as

$$
\begin{aligned}
\rho_{w} U^{*} \frac{\partial U^{*}}{\partial x_{*}} & =\rho_{w} \lambda \alpha \frac{\mathrm{d} A_{1}}{\mathrm{~d} x_{*}} Y_{*}^{\alpha}+\cdots, \\
\rho_{w} V^{*} \frac{\partial U^{*}}{\partial Y_{*}} & =-\rho_{w} \lambda \frac{\mathrm{d} A_{1}}{\mathrm{~d} x_{*}} Y_{*}^{\alpha}+\cdots, \\
\mu_{w} \frac{\partial^{2} U^{*}}{\partial Y_{*}^{2}} & =\alpha(\alpha-1)(\alpha-2) A_{1} Y_{*}^{\alpha-3}+\cdots .
\end{aligned}
$$

We see that the convective terms remain dominant provided that $\alpha>0$, in which case the momentum equation $(2.10 a)$ reduces at

$$
O\left(Y_{*}^{\alpha}\right): \quad(\alpha-1) \frac{\mathrm{d} A_{1}}{\mathrm{~d} x_{*}}=0 .
$$


Since the initial condition (2.12) does not contain any terms except the one which matches with the leading order term in (A 4), we have to conclude that

$$
A_{1}(-\infty)=0 \text {. }
$$

We see that a non-trivial solution for $A_{1}$ exists only if $\alpha=1$. Function $A_{1}\left(x_{*}\right)$ remains arbitrary in the framework of the asymptotic analysis of equations $(2.10 a)$. We, of course, expect that this function will be found as a part of the solution of the viscous-inviscid interaction problem as a whole.

Redenoting $A_{1}\left(x_{*}\right)$ as $A\left(x_{*}\right)$ renders (A 4$)$ in the form

$$
\Psi^{*}=\frac{1}{2} \lambda Y_{*}^{2}+A\left(x_{*}\right) Y_{*}+\cdots \quad \text { as } \quad Y_{*} \rightarrow \infty .
$$

\section{REFERENCES}

Benjamin, T. B. 1962 Theory of the vortex breakdown phenomenon. J. Fluid Mech. 14, 593-629.

Cattafester, L. N. \& Settles, G. S. 1992 Experimental on shock/vortex interaction. AIAA Paper 92-0315.

Chapman, D. R., Kuehn, D. M. \& Larson, H. K. 1956 Investigation of separated flows in supersonic and subsonic streams with emphasis on the effect of transition NACA Rep. 1356.

Crank, J. \& Nicolson, P. 1947 A practical method for numerical evaluation of solutions of partial differential equations of the heat-conduction type. Proc. Camb. Phil. Soc. 43, 50-67.

Daniels, P. 1974 Numerical and asymptotic solutions for the supersonic flow near the trailing edge of a flat plate. Q. J. Mech. Appl. Maths 27, 175-191.

Delery, J. M., Horowitz, E., Leuchter, O. \& Solignac, J. L. 1984 Fundamental studies of vortex flows. Rech. Aerospat. 2, 1-24.

Delery, M. 1994 Aspects of vortex breakdown. Progr. Aerospace Sci. 30, 1-59.

FErri, A. 1939 Atti di Guidonia No. 17. Engl. transl. 1940. Experimental results with airfoils tested in the high-speed tunnel at Guidonia. NACA TM 946.

GLotov, G. F. 1989 Interference of vortex braid with free-stream shock waves and nonisobatic jets. Uch. Zap. TsAGI 20 (5), 21-32.

Goldstern, S. 1930 Concerning some solutions of the boundary layer equations in hydrodynamics. Proc. Camb. Phil. Soc. 26 (1), 1-30.

Gustintsev, Y. A., Zelentsov, V. V., Ilyukhin, V. S. \& Pohkil, V. S. 1969 Structure of underexpended supersonic swirling gas jet. Izv. Akad. Nauk SSSR, Mekh. Zhid. Gaza (4), $158-162$.

Hall, M. G. 1972 Vortex breakdown. Annu. Rev. Fluid Mech. 4, 195-218.

Ivanyushin, A. K., Korotkov, Y. V. \& Nikolayev, A. V. 1989 Some aspects of the interference of shock waves with aerodynamic wake behind a body. Uch. Zap. TsAGI 20 (5), 33-42.

Kalkhoran, I. M. 1994 Vortex distortion during vortex-surface interaction in a Mach 3 stream. AIAA J. 32, 123-129.

Kalkhoran, I. M. \& Sforza, P. M. 1994 Airfoil pressure measurements during oblique shock wave-vortex interaction in a Mach 3 stream. AIAA J. 32, 783-788.

Kalkhoran, I. M., Smart, M. K. \& Betti, A. 1996 Interaction of a supersonic wing tip vortex with a normal shock. AIAA J. 34, 1855-1861.

Kalkhoran, I. M., Smart, M. K. \& Wang, F. Y. 1998 Supersonic vortex breakdown during vortex/cylinder interaction. J. Fluid Mech. 369, 351-380.

Korolev, G. L. 1989 On the theory of flow separation at the trailing edge of a thin profile. Izv. Akad. Nauk SSSR, Mekh. Zhid. Gaza (4), 55-59.

Korolev, G. L., GaJjar, J. S. B. \& Ruban, A. I. 2002 Once again on the supersonic flow separation near a corner. J. Fluid Mech. 463, 173-199.

Leibovich, S. 1978 The structure of vortex breakdown. Annu. Rev. Fluid Mech. 10, 221-246.

LeIBOvich, S. 1984 Vortex stability and breakdown: Survey and extension. AIAA J. 22, 1192-1206.

Lighthill, M. J. 1953 On boundary layers and upstream influence. II. Supersonic flows without separation. Proc. R. Soc. Lond. A 217, 478-507. 
Lighthill, M. J. 2000 Upstream influence in boundary layers 45 years ago. Phil. Trans. R. Soc. Lond. A 358, 3038-3061.

MAHESH, K. 1996 A model for the onset of breakdown in an axisymmetric compressible vortex. Phys. Fluids 8, 3338-3345.

Messiter, A. F. 1970 Boundary-layer flow near the trailing edge of a flat plate. SIAM J. Appl. Maths 18, 241-257.

Metwally, O., Settles, G. S. \& Horstman, C. 1989 An experimental study of shock/vortex interaction. AIAA Paper 89-0082.

NeILAND, V. Y. 1969 Theory of laminar boundary layer separation in supersonic flow. Izv. Akad. Nauk SSSR, Mekh. Zhid. Gaza (4), 53-57.

Prandtl, L. 1904 Über flüssigkeitsbewegung bei sehr kleiner Reibung. In Verh. III. Intl Math. Kongr., Heidelberg, pp. 484-491. Teubner, Leipzig, 1905.

Reyhner, T. A. \& FLÜGge-LotZ, I. 1968 The interaction of a shock wave with a laminar boundary layer. Intl J. Nonlinear Mech. 3, 173-199.

SARPKAYA, T. 1971 On stationary and travelling vortex breakdowns. J. Fluid Mech. 45, 585-595.

SMART, M. K. \& Kalkhoran, I. M. 1995 The effect of shock strength on oblique shock-vortex interaction. AIAA J. 33, 2137-2143.

Smart, M. K. \& KalKhoran, I. M. 1997 Flow model for predicting normal shock wave induced vortex breakdown. AIAA J. 35, 1589-1596.

Stewartson, K. \& Williams, P. G. 1969 Self-induced separation. Proc. R. Soc. Lond. A 312, 181-206.

SycheV, VIC. V. 1978 Breakdown of a plane laminar wake. Uch. Zap. TsAGI 9, 9-16.

SycheV, V. V. 1972 Laminar separation. Izv. Akad. Nauk SSSR, Mekh. Zhid. Gaza (3), 47-59.

Sychev, V. V., Ruban, A. I., Sychev, Vic. V. \& Korolev, G. L. 1998 Asymptotic Theory of Separated Flows. Cambridge University Press.

Zataloka, V. V., Ivanyushinin, A. K. \& NikolayeV, A. V. 1978 Interference of vortices with shocks in airscoops. dissipation of vortices. Fluid Mech. - Sov. Res. 7, 135-158. 